\title{
PRINCIPIOS IMPLÍCITOS Y RELACIONES SISTEMÁTICAS *
}

\author{
Juan Luis Moreno** \\ Universidad de Buenos Aires
}

RESUMEN. Este artículo hace un examen formal del razonamiento jurídico con principios implícitos desde el positivismo lógico y partiendo de los trabajos de ALONSO en la materia. A la vista de las tesis de la separación fuerte y débil entre principios y reglas y de las diferencias entre norma y proposición normativa, se presentan problemas para considerar que las consecuencias lógicas que ALONSO denomina principios implícitos son principios. Como correlación caso-solución carecen de la nota de generalidad y como relaciones de preferencia son sólo proposiciones normativas que derivan de aplicar un criterio de preferencia al sistema normativo. En su caso, el criterio de preferencia podría considerarse un principio implícito, pero al ser una proposición normativa surgen problemas de juridicidad, justificación y coherencia del principio.

Palabras clave: Principios implícitos, normas, proposiciones normativas, lógica de preferencias.

\section{Implicit Principles and Systematics Relations}

ABSTRACT. This paper reviews legal reasoning with implicit principles from the formal perspective of the logical legal positivism and taking as starting point the works by ALONSO on the subject. In view of the strong and weak demarcation thesis between principles and rules and the differences between norms and norm-propositions, it concludes that the logical consequences considered implicit principles by ALONSO are not principles. As case-solution correlation they lack the required note of generality and as preference relations they are only norm-propositions derived from the application of a preference criterion to the normative system. If any, the preference criterion could be understood as the implicit principle, however, in so far as the criterion is a norm-proposition, certain concerns arise as to the legal character of the principle, its justification and coherence.

Keywords: Implicit principles, norms, norm-propositions, preference logic.

* Fecha de recepción: 18 de diciembre de 2014. Fecha de aceptación: 9 de marzo de 2015.

** El autor agradece los comentarios de Juan Pablo ALONSO y Gabriela SCATAGLINI y, muy especialmente, la constante ayuda, sugerencias y ánimos de Miranda DEL CORRAL. Este trabajo se incardina dentro del proyectos DeCyT DCT1403 sobre principios jurídicos implícitos. 


\section{INTRODUCCIÓN}

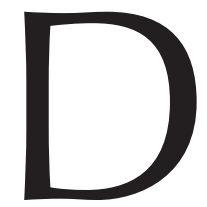

esde mediados del siglo xx, el positivismo jurídico ha tratado con gran atención la aplicación de la lógica al derecho y el papel de los principios en los sistemas jurídicos. Aunque el debate sobre la aplicación de la lógica al derecho ya se había planteado en épocas pasadas ${ }^{1}$, en su versión moderna se inició con la publicación en 1951 del artículo «Deontic Logic» de VON WRIGHT y fue impulsado por trabajos de Ross (Directives and Norms), del propio VON WRIGHT (Norm and Action) o de AlCHOURRÓn y BulYGIN (Normative Systems) ${ }^{2}$. Este debate sigue vivo, como manifiesta la reciente aparición de la obra Deontic Logic and Legal Systems de NAVARRO y RODRÍGUEZ ${ }^{3}$. Algo parecido sucede con el debate en la Filosofía del derecho sobre el papel de los principios en los sistemas jurídicos y su uso en la argumentación jurídica. La cuestión ya atrajo interés de los teóricos del derecho en épocas cercanas a la codificación ${ }^{4}$, pero el punto de partida del debate moderno sobre los principios se sitúa en 1967 con la aparición del artículo de DwORKIN «The Model of Rules» posteriormente incluido en el libro Taking Rights Seriously ${ }^{5}$, momento desde el cual se ha dedicado una atención creciente al tema.

Estas dos grandes áreas de debate no han permanecido aisladas. Teóricos como RAZ $^{6}$, ALEXY $^{7}$ o AARNIO ${ }^{8}$ han apelado a la lógica en mayor o menor medida para tratar los principios jurídicos y su diferencia con las reglas. Entre los autores hispanohablantes, Juan Pablo ALONSO ha prestado particular atención al análisis lógico de los principios implícitos desde una concepción positivista ${ }^{9}$. ALONSO emplea el sistema de lógica deóntica desarrollado por ALCHOURRÓN y BULYGIN y el concepto de coherencia de MACCORMICK como adecuación de un sistema normativo a uno o varios principios ${ }^{10}$. La combinación de ambas posturas permite a ALONSO, mediante un elegante procedimiento, extraer de un sistema normativo consecuencias lógicas que son formuladas bien como normas de mandato entendidas como enunciados que conectan un caso genérico con una solución normativa, o bien como relaciones que explicitan el orden de preferencia de las soluciones normativas del sistema según sus propiedades relevantes. Las relaciones de preferencia se emplean para crear diagramas gráficos que presentan con claridad las relaciones entre normas y para evaluar la coherencia del sistema y sugerir correcciones a problemas lógicos del sistema como lagunas normativas $\mathrm{y}$ antinomias.

La intención de este trabajo es señalar que las consecuencias lógicas y relaciones enunciadas por ALONSO no presentan las características distintivas de los principios jurídicos, por lo que dentro de los planteamientos empleados por AlONSO existirían

1 Vid. HAACK, 2007: 1-3.

2 Von Wright, 1963; Ross, 1968, y Alchourrón y Bulygin, 1974. Para una revisión histórica de la aplicación de la lógica al derecho, vid. HAACK, 2007: 9-14.

3 NaVArro y RodrígueZ, 2014.

4 Prieto SAnchís, 1992: 17-20; AtienZa y Ruiz Manero, 1996: 1-6.

5 DWORKIN, 1978.

6 RAZ, 1972.

7 Alexy, 1989, 1993, y 2000.

8 AARNIO, 2011.

9 Vid. Alonso, 1995, 2012 y 2013.

10 Alonso, 2013: 357. 
dificultades para considerarlas principios jurídicos implícitos del sistema. Para ello, como punto de partida expondré en el apartado siguiente las características que se han atribuido a los principios jurídicos desde las dos grandes posturas acerca de su naturaleza, esto es, las tesis de la separación fuerte y débil. Después examinaré en el apartado 3 las características particulares que se atribuye a los principios implícitos como subgénero de los principios, para lo cual expondré la distinción entre lenguaje objeto y metalenguaje a la que acudiré repetidas veces en la exposición. Una vez examinados estos conceptos, expondré brevemente en el apartado 4 los trabajos de ALONSO y comprobaré si las características diferenciales de los principios jurídicos están presentes en las consecuencias lógicas y relaciones que enuncia, separando entre generalidad en el apartado 5 y peso en el apartado 6. En la medida que, como anticipé, no es posible hallar tales características, en el apartado 8 propondré una interpretación diferente que afecta a las relaciones entre normas enunciadas, analizaré algunas consecuencias de la propuesta en el apartado 9 y elaboraré las conclusiones en el apartado 10. Por último, el apartado 11 incluye un anexo que explicita las relaciones entre normas en los sistemas normativos analizados por ALONSO.

\section{EL PESO Y LA GENERALIDAD DE LOS PRINCIPIOS JURÍDICOS}

Las teorías que analizan las diferencias entre reglas y principios jurídicos se dividen en la tesis de la diferenciación fuerte, lógica o estricta y la de la separación débil ${ }^{11}$. La tesis de la separación fuerte fue la primera en surgir. Su formulación original se debe a DwORKIN, quien en su trabajo «The Model of Rules» ${ }^{12}$ consideró que los principios y las reglas son clases diferentes de normas y que entre ambas se puede trazar una distinción tajante de carácter lógico contraponiendo sus características respectivas y sus usos en la práctica y razonamiento jurídicos. Según la tesis de la separación fuerte: i) los principios presentan una característica inconmensurable denominada generalidad, peso, vaguedad o apertura, que no existe en las normas; ii) los principios y las reglas colisionan entre sí de diferente forma; iii) principios y reglas tienen diferentes usos en la argumentación jurídica, y iv) principios y reglas tienen también diferente consideración como razones para la acción ${ }^{13}$. De estos criterios, me centraré fundamentalmente en el análisis de la característica de peso, vaguedad, apertura o generalidad, puesto que será la característica que se usará para analizar los trabajos de ALONSO. Esta característica ha sido examinada desde dos perspectivas diferentes. La primera considera que la característica se manifiesta en la mayor generalidad o apertura que tienen los principios frente a las reglas al configurar las condiciones de aplicación o las conductas exigidas por el principio. La segunda considera que esa característica es más evanescente y la trata como una dimensión de peso o importancia de la que las reglas carecen.

Cuando se entiende como generalidad o apertura, la característica diferencial de los principios jurídicos se relaciona unas veces con las condiciones de aplicación del principio y otras con las conductas que exige. Por ejemplo, DwORKIN la refirió a las

11 Vid. Prieto Sanchís, 1992: 32-64, y Alonso, 2006: 164-178.

12 Vid. nota 5 anterior.

13 Unos criterios similares se mencionan en AARNIO, 1997: 23-24, y 2011: 120. 
condiciones de aplicación ${ }^{14}$ y RAZ a las acciones prescritas, que serían altamente genéricas $^{15}$. Los trabajos de ALEXY sobre teoría de la argumentación aludían a la generalidad de los principios como falta de concreción de las condiciones de aplicación ${ }^{16}$ y en la doctrina latina, ATIENZA y RUIZ MANERO han distinguido dos subclases dentro de los principios (principios en sentido estricto y directrices o normas programáticas) ${ }^{17}$, ambas definidas por su generalidad: los principios en sentido estricto configuran el caso de forma abierta con propiedades relevantes que no están «siquiera genéricamente determinadas», y las directrices «configuran de forma abierta tanto sus condiciones de aplicación como el modelo de conducta prescrito» ${ }^{18}$.

En la segunda perspectiva, la característica diferencial entendida como peso se vincula a la colisión entre principios. Según DWORKIN, el peso era usado para resolver las colisiones entre principios. La presencia del peso en los principios permitía que el principio de mayor peso desplazara al de menor sin que ninguno de ellos fuera expulsado del sistema. Por el contrario, las normas carecían de dimensión de peso, por lo que se aplicaban todo-o-nada y en caso de conflicto no podían coexistir en el sistema, por tanto una expulsaba a la otra ${ }^{19}$.

Posteriormente AlEXY aclaró el tratamiento lógico del peso. Para AleXY los principios jurídicos son mandatos de optimización ${ }^{20}$, es decir, normas «que ordenan que algo sea realizado en la mayor medida posible, dentro de las posibilidades jurídicas y reales existentes», por lo que «pueden cumplirse en diferentes grados» frente a las reglas, que sólo pueden ser cumplidas o no. ALEXY ha propuesto tratar el peso de los principios como una preferencia entre diferentes estados ideales del mundo y aplicar la lógica de preferencias sobre soluciones normativas ${ }^{21}$. Esta solución ha sido también aceptada por AARNIO ${ }^{22}$ y NAVARRO y RODRÍGUEZ ${ }^{23}$, de modo que siguiendo a estos tres autores consideraré que el tratamiento lógico del peso es posible si se acude a la lógica de preferencias, pero que no sería posible mediante la correlación caso-solución.

14 «A principle like "No man may profit from bis own wrong" does not even purport to set out conditions that make its application necessary», DWORKIN, 1978: 26.

15 RAZ, 1972: 828 y 838. Pese a mantener la distinción lógica entre principios y normas, RAZ reconoce que es difícil trazar un límite claro puesto que se trata de una diferencia de grado que permite muchas zonas grises (838). De hecho, en atención a sus trabajos sobre razón práctica y normas, AARNIO incardina a RAZ dentro de la tesis de la separación débil (vid. AARNIO, 2011: 120). Es también característico de la concepción de los principios sostenida por RAZ el no considerar que las alusiones resumidas a varias normas sean principios, sino meras formas de enunciación abreviada y entender que los principios pueden justificar normas pero las normas no pueden justificar principios (828 y 839).

16 «Principles are normative propositions of such a high level of generality that they can as a rule not be applied without the addition of further normative premisses and are usually subject to limitation on account of other principles», AlEXY, 1989: 260. La misma idea aparece en la nota 81 en p. 243.

17 ATIENZA y RUIZ MANERO, 1996: 5 y ss. AARNIO también se realiza una división adicional entre principios, principios como reglas, reglas como principios y reglas (AARNIO, 1997, y 2011: 121-123).

18 ATIENZa y Ruiz MANERO, 1996: 9-11.

19 DWORKIN, 1978: 22-26.

20 Atienza y RUIZ MANERo aplican esta descripción a la forma en que operan las directrices. Vid. AtiENZA y RuIZ MANERO, 1996: 11.

21 AleXy, 1993: 86-87 y 90-98, y 2000: 195, 299 y 300. También en esta línea, PeCZENIK, 1992.

22 AARNIO, 2011: 123.

23 NAVArRo y Rodríguez, 2014: 194-195. Como alternativa, Prieto relaciona la idea de peso con el criterio de especialidad. Vid. PRIETO SANCHís, 1992: 43. 
La tesis de la separación estricta ha sido criticada alegando que ofrece una diferencia más aparente que real no verificada en la práctica. Como alternativa se ha propuesto la tesis de la separación débil, según la cual entre principios y normas no existe una separación de carácter lógico, sino que hay sólo una relación de grado que se manifiesta al contraponer diferentes normas y configurar unas de ellas como principios y otras como reglas. En cualquier caso, los autores que postulan la separación débil aluden a los mismos conceptos de generalidad y peso. COMANDUCCI considera que al contraponer diferentes normas unas se configurarán como principios y otras como reglas según cual de las contrapuestas presente en mayor medida la dimensión inconmensurable de apertura o peso ${ }^{24}$. AARNIO sostiene que, en las actividades de los operadores jurídicos, a los principios les corresponde el peso y ponderación ${ }^{25}$, peso expresado como se dijo mediante lógica de preferencias ${ }^{26}$. PRIETO alude a la apertura de la condición de aplicación entre las normas que se comparen ${ }^{27}$ y, por último, ALCHOURRÓN y BULYGIN apelan al mayor grado de generalidad de los principios, aunque no tratan específicamente las diferencias entre principios y reglas ${ }^{28}$.

En conclusión, la característica distintiva de los principios frente a las reglas se puede entender como mayor generalidad o como presencia de peso. Si se entiende como generalidad, esta característica se vincula a la configuración abierta de las condiciones de aplicación o de las conductas exigidas por una norma, lo que para aproximar esta generalidad a la nomenclatura de ALCHOURRÓN y BULYGIN que sigue AlONSO se podría representar como configuración de las propiedades del caso, lo cual en la terminología del Normative Systems equivale a decir que un principio configura un caso menos fino que la regla frente a la que se contrapone para calificarse como principio $^{29}$. Si se entiende como peso, la característica diferencial significaría que los principios expresan una preferencia entre dos estados ideales del mundo que se representaría mediante la lógica de preferencias siguiendo a ALEXY, AARNIO o RODRÍGUEZ y NAVARRO.

\section{LOS PRINCIPIOS IMPLÍCITOS Y LAS PROPOSICIONES NORMATIVAS}

Además de las características propias de los principios frente a las reglas, la doctrina atribuye a los principios implícitos ciertas características particulares que será preciso tener en cuenta para examinar los trabajos de AlONSO. Para exponer tales peculiaridades me apoyaré en la exposición de los principios implícitos que realiza PRIETO por ser particularmente clara.

PRIETO trata los principios implícitos como un subtipo de principios jurídicos. Con apoyo en las clasificaciones de WROBLEWSKI y GUASTINI, divide los principios jurídicos en tres categorías según su respaldo institucional: principios explícitos, prin-

24 COMANDUCCI, 1998: 93-95.

25 AARNIO, 2011: 119-124.

26 Ibid., 123. Esta postura se asemeja a la sostenida por RAZ en 1972, aunque RAZ alude expresamente a la existencia de una distinción lógica.

27 PRIETO SANCHÍs, 1992: 39 y 56-57.

28 AlchOURRÓn y BulYgin, 1974: 140-141.

29 Ibid., $140-141$ y $148-152$. 
cipios implícitos y principios extrasistémicos ${ }^{30}$. De estas categorías, considera que principios los explícitos son aquellos que están plasmados expresamente en el derecho positivo; los implícitos o inexpresos pero implícitos son aquellos que pueden extraerse de un sistema normativo mediante inducción o deducción seguida de inducción ${ }^{31} ; \mathrm{y}$, por último, los principios extrasistémicos o totalmente inexpresos son aquellos tienen un origen doctrinal más que jurisprudencial y una elaboración mucho más compleja que descansa en una argumentación con base política o moral ${ }^{32}$.

En todo caso, PRIETO hace una reflexión de gran importancia para analizar los principios implícitos desde un punto de vista formal. Este autor reconoce que la distinción entre principios extrasistémicos y principios implícitos tiene fronteras poco nítidas porque los principios implícitos también tendrían un aspecto de elaboración por el intérprete que los desarrolla, en tanto que la selección de los aspectos relevantes para la inducción de principios implícitos conllevaría necesariamente «elecciones u opciones valorativas», es decir, «ingredientes extrasistemáticos» ${ }^{33}$. Una reflexión parecida hacen ATIENZA y RUIZ MANERO, para los cuales los principios implícitos están también conectados con la prevalencia encubierta en el ordenamiento jurídico de intereses o valores ${ }^{34}$.

La mención a ingredientes extrasistemáticos o extrasistémicos se puede examinar con apoyo en la distinción lógica entre lenguaje objeto y metalenguaje, que se relaciona en lógica deóntica con la distinción entre normas y proposiciones normativas. Siguiendo a ALCHOURRÓN y BULYGIN, «[1] as normas son enunciados prescriptivos que se usan para ordenar, prohibir o permitir conductas humanas. Las proposiciones normativas son enunciados descriptivos que se usan para informar acerca de las normas o acerca de las obligaciones, prohibiciones o permisiones establecidas por las normas». De esta manera, cuando se enuncia una proposición sobre un sistema, «en lugar de formular una norma, enunciamos una proposición normativa; el enunciado que expresa esta proposición pertenece a un nivel lingüístico distinto que los enunciados (que expresan normas) del sistema» ${ }^{35}$.

Cuando PRIETO o AtIEnZa y RuIZ MANERO aluden a que los principios implícitos incorporan algún tipo de valor como elemento extrasistémico, parecen asumir que la argumentación sobre principios implícitos se sitúa en un nivel superior del discurso. Las normas de un sistema normativo pertenecen al lenguaje objeto y son enunciados prescriptivos de acciones. Sin embargo, al incorporar un elemento extrasistémico para realizar una elaboración sobre las normas del sistema, la elaboración producirá pro-

30 PRIETO SANCHÍs, 1992: 134.

31 Ibid. Los principios implícitos se extraen por inducción «a partir de una o varias disposiciones que se supone constituyen casos de aplicación o especificación de dicho principio. En cierto modo, cabe pensar que estamos ante lo que Alchourrón y Bulygin han llamado la reformulación del sistema». Los principios se extraen por deducción seguida de inducción a partir de principios más generales «o inductivamente a partir de una serie de preceptos» supuestamente confirmadores de su existencia. En este último caso se trataría de principios «reconocidos por la doctrina y aplicados por los operadores jurídicos» pero «no [...] expresamente recogidos en ninguna disposición».

32 AtienZa y Ruiz Manero, 1996: 18-19.

33 PRIETO SANCHÍS, 1992: 141-143.

34 AtienZa y Ruiz MANERO, 1996: 18-19.

35 AlchourRón y Bulygin, 1974: 173. 
posiciones normativas, esto es, enunciados descriptivos de las normas del sistema que pertenecen a un nivel superior del lenguaje y carecen de carácter prescriptivo.

La aceptación de elementos extrasistémicos en la argumentación jurídica plantea dos problemas. En primer lugar, para que las elaboraciones con base en elementos extrasistémicos sean principios deberán ser normas y tener generalidad o peso. Sin embargo, las elaboraciones con base en elementos extrasistémicos son proposiciones normativas, pertenecen a un nivel diferente del lenguaje al de las normas del sistema y no pueden contraponerse a las reglas del sistema para calificarse como principios en atención a su generalidad o peso. Por otro lado, al considerar que los principios son proposiciones normativas, los principios no pertenecerán a un sistema, por lo que no serán principios implícitos del sistema ni principios jurídicos, puesto que las proposiciones normativas no son prescriptivas.

\section{EL TRATAMIENTO DE LOS PRINCIPIOS IMPLÍCITOS POR ALONSO}

Para exponer su concepción lógico-positiva de los principios implícitos, ALONSO ha acudido a diversos ejemplos del derecho privado. Entre ellos, ha empleado: i) el sistema que establece el régimen de reintegro de gastos tras la restitución de la posesión de inmuebles a su titular según los arts. 453 a 455 del Código Civil español y que denominaré $\mathrm{S} 1$ en lo sucesivo ${ }^{36}$; ii) el sistema que contiene el régimen de la reivindicación de inmuebles propiedad de tercero en el anteproyecto de Código Civil brasileño elaborado por FREITAS al que denominaré S2, y iii) el sistema que regula el mismo régimen de reivindicación en el Código Civil argentino vigente hasta 2016 y que denominaré S3 ${ }^{37}$.

\subsection{S1: régimen de reintegración de gastos tras restitución de inmueble}

El sistema S1 tiene siete normas y, para expresarlas, ALONSO emplea las abreviaturas que se detallan a continuación. Las propiedades relevantes cuya concurrencia configura los casos a los que se aplica el resultado normativo determinado por la norma se expresan mediante letras mayúsculas. Las propiedades relevantes son: GN: gastos necesarios; GU: gastos útiles; GS: gastos suntuarios; y BF: buena fe. En cuanto a los resultados normativos, los operadores deónticos son los habituales y se expresan comenzando en mayúscula: O: obligatorio; Ph: prohibido; F: facultativo. Las acciones a las que se aplican dichos operadores deónticos se expresan mediante letras minúsculas y son los siguientes: p significa pagar gastos; q significa abonar aumento de valor; y s significa derecho a separar el bien resultante del gasto realizado, con derecho a una acción adicional, bien $\mathrm{p}$ que significa recibir gastos — $\mathrm{s}(\mathrm{p})$ - o bien q que significa recibir aumento de valor $-\mathrm{s}(\mathrm{q})-$. La negación se expresa mediante el símbolo $\neg$ que se lee «no», la conjunción entre propiedades relevantes o soluciones normativas se expresa mediante el símbolo \& que se lee «y», y la disyunción se expresa mediante el símbolo $\vee$ que se lee «o» y se entiende como disyunción excluyente.

\footnotetext{
36 AlONSO, 1995.

37 S2 y S3 se extraen de AlCHOuRrón y Bulygin, 1974: 38 y ss.
} 
Por último, las normas se expresan como correlación entre conjuntos de propiedades relevantes (que configuran el caso) y una o varias acciones precedidas por un operador deóntico (que describe la solución normativa de la norma). La correlación se expresa con el símbolo $\rightarrow$ que se lee «si $[. .$.$] entonces [. .]$.$» . Por razones de espacio,$ en las matrices se emplea una notación donde la solución normativa aparece en primer lugar seguida del caso, ambas conectadas con el símbolo / que se lee «siempre que».

De acuerdo con las abreviaturas y simbología explicadas, las normas del sistema S1 son N1: GN $\rightarrow$ Op; N2: GN\&BF $\rightarrow$ Fr; N3: GU\&BF $\rightarrow$ OpvOq; N4: GU\&BF $\rightarrow$ Fr; N5: Gs\&BF $\rightarrow$ Fs (p); N6: GN\& $\neg \mathrm{BF} \rightarrow \mathrm{Op}$; y N7: GS\& $\neg \mathrm{BF} \rightarrow \mathrm{Fs}(\mathrm{q})$, con las que AlONSO construye la siguiente matriz:

\section{S1 ${ }^{38}$}

\begin{tabular}{|c|c|c|c|c|c|c|c|c|c|c|c|}
\hline & GN & GU & GS & BF & $\mathrm{Op} / \mathrm{GN}$ & $\mathrm{Fr} / \mathrm{GN} \& \mathrm{BF}$ & $\mathrm{Op \vee Oq} / \mathrm{GU} \& \mathrm{BF}$ & $\mathrm{Fr} / \mathrm{GU} \& \mathrm{BF}$ & $\mathrm{Fs}(\mathrm{p}) / \mathrm{Gs} \& \mathrm{BF}$ & $\mathrm{Op} / \mathrm{GN} \& \neg \mathrm{BF}$ & Fs $(q) / G S \& \neg B F$ \\
\hline 1 & + & - & - & + & Op & $\mathrm{Fr}$ & & & & & \\
\hline 2 & + & - & - & - & Op & & & & & Op & \\
\hline 3 & - & + & - & + & & & $\mathrm{Op} \vee \mathrm{Oq}$ & $\mathrm{Fr}$ & & & \\
\hline 4 & - & + & - & - & & & & & & & \\
\hline 5 & - & - & + & + & & & & & $\mathrm{Fs}(\mathrm{p})$ & & \\
\hline 6 & - & - & + & - & & & & & & & $\mathrm{Fs}(\mathrm{q})$ \\
\hline
\end{tabular}

De esta matriz del sistema S1, ALONSO extrae los siguientes principios implícitos:

i) Como correlación caso-solución extrae el principio de que «los gastos no suntuarios de buena fe tiene[n] derecho de retención», principio $\alpha$ expresado como $(\neg \mathrm{GS} \& \mathrm{BF}) \rightarrow \mathrm{Fr}$ o, en notación alternativa, $\mathrm{Fr} /(\neg \mathrm{GS} \& \mathrm{BF})^{39}$.

ii) Como relación de orden de preferencias entre soluciones normativas para cada propiedad relevante, según los criterios $\mathrm{Op} \geq \mathrm{Op} \vee \mathrm{Oq}(\mathrm{Op} \vee \mathrm{Oq}) \& \mathrm{Fr}>\mathrm{Fs}(\mathrm{p})$ y $\mathrm{O} p \geq \mathrm{Fs}(\mathrm{q})^{40}$, extrae el principio $\beta$ relativo al tratamiento de los gastos según el que «el

38 En principio, cuatro propiedades relevantes darían lugar a $2 \mathrm{n}=24=16$ casos. No obstante, ALONSO elimina los casos en que concurren dos de las propiedades GN, GU o GS o las tres porque las propiedades no son independientes (no representan casos individuales) y la clasificación de gastos es conjuntamente excluyente y exhaustiva, es decir, cada gasto puede ser clasificado solamente dentro de una categoría (necesario, útil o suntuario) y las tres categorías agotan todos los gastos posibles.

39 AlONSO, 1995: 417.

40 Esta fórmula expresa mediante lógica de relaciones el criterio de ALONSO en ALONSO, 1995: 417-418: «¿Cuándo un poseedor tiene mejor derecho que otro poseedor? En algunas ocasiones se trata del derecho a un reintegro más oneroso. En otras ocasiones se trata del derecho accesorio de retención que algunos poseedores tienen y otros carecen. [...] El derecho a percibir el costo (Op: caso 1) es mejor que el derecho a percibir el costo o el mayor valor (OpvOq: caso 3) ya que al tener el deudor la alternativa entre p o q, siempre puede elegir la menos onerosa, que en general será el abono del mayor valor (q). A su vez, los casos 1 y 3 tienen el mismo derecho de retención. El derecho de 1 en algunas ocasiones es mejor y en otras es igual que el de 3 . El derecho a $[(\mathrm{Op} \vee \mathrm{Oq}) \& \mathrm{Fr}$ : caso 3$]$ es mejor o igual que el ius tollendi $[\mathrm{Fs}(\mathrm{p})$; caso 5]. En cuanto a los bienes no separables es mejor, ya que 3 tiene derecho a reintegro y 5 carece de derecho a reintegro alguno. En cuanto a los bienes separables el derecho es similar, ya que el ius tollendi es un derecho de retención sobre un objeto separable, ya que el anterior poseedor debe entregar el objeto si el nuevo poseedor decide abonárselo. [...] En cuanto a los GN, el caso 1 tiene un derecho de retención del que el caso 2 carece. Pero este último tiene derecho a un reintegro (p) similar a 1. En cuanto a los GS, el caso 5 tiene el Fs (p), que es mejor o igual derecho que el Fs (q) 
derecho de quien realizó un gasto necesario es mejor o igual que el de quien realizó un gasto útil, y éste mejor o igual que el de quien realizó un gasto suntuario», expresado como $\mathrm{GN} \geq \mathrm{GU} \geq \mathrm{GS}^{41}$; $y$ también extrae el principio $\gamma$ relativo al tratamiento normativo de la buena fe, según el cual «[e]l derecho del anterior poseedor de buena fe (D BF), es mejor o igual que el derecho del anterior poseedor de mala fe $(\mathrm{D} \neg \mathrm{BF}) »$, principio que expresa como $\mathrm{BF}>\neg \mathrm{BF}^{42}$.

\subsection{S2: régimen de reintegración de inmueble ajeno (proyecto de FrEITAS)}

ALONSO ha reconstruido en posteriores trabajos ${ }^{43}$ el sistema S2 de reintegración de inmueble ajeno según el proyecto de Código Civil brasileño de FrEITAS. La notación es la misma que en S1, pero las abreviaturas de propiedades relevantes son BFE: buena fe del enajenante; BFA: buena fe del adquirente; y TO: título oneroso, y la única acción de las soluciones normativas en $\mathrm{S} 2$ es r: restituir inmueble. El sistema incluye las normas N1: $\neg \mathrm{BFE} \rightarrow$ Or; N2: $\neg \mathrm{BFA} \rightarrow \mathrm{Or}$; N3: $\neg \mathrm{TO} \rightarrow$ Or; y N4: BFE\&BFA\&TO $\rightarrow$ Fr:

S2

\begin{tabular}{|c|c|c|c|c|c|c|c|}
\hline & BFE & BFA & TO & $\mathrm{Or} / \neg \mathrm{BFE}$ & Or/ $/$ BFA & Or/ᄀTO & Fr/BFE\&BFA\&TO \\
\hline 1 & + & + & + & & & & $\mathrm{Fr}$ \\
\hline 2 & - & + & + & Or & & & \\
\hline 3 & + & - & + & & Or & & \\
\hline 4 & - & - & + & Or & Or & & \\
\hline 5 & + & + & - & & & Or & \\
\hline 6 & - & + & - & Or & & Or & \\
\hline 7 & + & - & - & & Or & Or & \\
\hline 8 & - & - & - & Or & Or & Or & \\
\hline
\end{tabular}

De esta matriz del sistema S2, ALONSO extrae los siguientes principios implícitos como relación de orden de preferencias según el criterio $\mathrm{FR}>\mathrm{OR}^{44}$ :

i) $\delta$ : BFE $\geq \neg B F E$ : «Quien adquiere de un enajenante de buena fe tiene mejor o igual derecho que quien adquiere de enajenante de mala fe, siempre y cuando el resto de las circunstancias se mantengan constantes».

del 6. En algunas ocasiones es mejor, tal como es el derecho al costo de la mejora (p), y en otras ocasiones es igual, como lo es en el caso de los bienes no separables».

41 AlONSO, 1995: 417.

42 Ibid., 1995: 418.

43 Ibid., 2013.

44 Ibid., 2013: 361. La explicitación del criterio es la siguiente: «Los principios implícitos establecen la relación de "tener un derecho mejor o igual que", relación que, en este contexto, sólo significa que "Facultativo Restituir (FR)" es un derecho mejor que "Obligatorio Restituir (OR)", toda vez que quien puede conservar el inmueble (FR) está mejor posicionado, en términos patrimoniales, que aquel que debe restituir el inmueble (OR) al reivindicante. Complementariamente, hay un derecho igual cuando ambos pueden conservar el inmueble (FR) o cuando ambos deben restituirlo (OR)». 
(ii) $\varepsilon$ : $\mathrm{BFA} \geq \neg \mathrm{BFA}:$ «Quien adquiere de buena fe tiene mejor o igual derecho que quien adquiere de mala fe, siempre y cuando el resto de las circunstancias se mantengan constantes».

(iii) $\zeta$ : $\mathrm{TO} \geq \neg \mathrm{TO}:$ : Quien adquiere a título oneroso tiene mejor o igual derecho que quien adquiere a título gratuito, siempre y cuando el resto de las circunstancias se mantengan constantes».

\subsection{S3: reintegración de inmueble ajeno en el Código Civil argentino}

Por último, ALONSO ha reconstruido también el sistema S3 sobre el régimen de reintegración de inmueble ajeno en el código civil argentino vigente hasta 2016, con la misma notación que en S2 y a partir de las normas N1: BFE\&BFA\&TO $\rightarrow$ Fr; $\mathrm{N} 2: \mathrm{BFE} \& \neg \mathrm{BFA} \rightarrow \mathrm{Or}$; y N3: $\neg \mathrm{TO} \rightarrow \mathrm{Or}$.

S3

\begin{tabular}{|c|c|c|c|c|c|c|}
\hline & BFE & BFA & TO & $\mathrm{Fr} / \mathrm{BFE} \& \mathrm{BFA} \& \mathrm{TO}$ & Or/BFE\& $\neg \mathrm{BFA}$ & $\mathrm{Or} / \neg \mathrm{TO}$ \\
\hline 1 & + & + & + & $\mathrm{Fr}$ & & \\
\hline 2 & - & + & + & & & \\
\hline 3 & + & - & + & & Or & \\
\hline 4 & - & - & + & & & \\
\hline 5 & + & + & - & & & Or \\
\hline 6 & - & + & - & & & Or \\
\hline 7 & + & - & - & & Or & Or \\
\hline 8 & - & - & - & & & Or \\
\hline
\end{tabular}

De esta matriz del sistema S3 ALONSO no extrae principios implícitos, sino que aplica los principios de S2 para colmar las lagunas normativas en los casos 2 y 4.

\section{PRINCIPIOS IMPLÍCITOS Y GENERALIDAD}

Según anticipado, la propuesta de este trabajo es que las relaciones $\alpha, \beta, \gamma, \delta, \varepsilon$ y $\zeta$ reveladas por ALONSO en S1 y S2 presentan inconvenientes para ser consideradas principios jurídicos implícitos cuando se analizan sus características lógicas desde una perspectiva positivista. Por ello, para verificar si su análisis lógico permite considerarlos o no principios implícitos, en los apartados siguientes examinaré si, al realizar un análisis formal, $\alpha, \beta, \gamma, \delta, \varepsilon$ y $\zeta$ presentan las notas de generalidad y peso que configuran a los principios cuando se contraponen a las normas del sistema consideradas reglas.

En primer lugar trataré los principios expresados como correlación caso-solución. A efectos de su análisis lógico, cuando los principios se enuncian como una relación entre un caso genérico y una solución normativa la notación lógica empleada es la lógica deóntica, no la lógica de preferencias. Por tanto, y según analizado en el apartado 2, la característica diferencial de los principios se manifiesta a efectos lógicos como mayor 
generalidad respecto de las reglas del sistema, y por tanto para su análisis formal se deberá obviar de momento el aspecto del peso cuyo tratamiento corresponde a la lógica de preferencias. Resulta de esta manera que para comprobar desde un punto de vista formal si las consecuencias lógicas enunciadas por ALONSO son principios, procederá verificar si, enunciados lógicamente como una correlación caso-solución, tienen la característica diferencial de los principios entendida como mayor generalidad, esto es, si configuran el caso como un caso menos fino que las restantes normas del sistema (que se entenderán como reglas).

De los principios analizados por ALONSO en los trabajos citados, el único principio enunciado como correlación caso-solución es el principio $\alpha$ de S1. El principio $\alpha$ dispone $\neg \mathrm{GS} \& \mathrm{BF} \rightarrow \mathrm{Fr}$. Para evaluar si $\alpha$ tiene suficiente generalidad como para ser considerado principio en $\mathrm{S} 1$ según sus características lógicas y en contraposición a las normas expresas del mismo, consideradas reglas, será necesario comprobar si $\alpha$ como correlación caso-solución configura el caso de manera más general o menos fina, esto es, si tiene en cuenta menos propiedades relevantes que las normas de S1.

Las normas de $\mathrm{S} 1$ (y entre paréntesis, el número de sus propiedades relevantes) son N1: GN $\rightarrow \mathrm{Op}(1)$; N2: $\mathrm{GN} \& \mathrm{BF} \rightarrow \mathrm{Fr}(2) ; \mathrm{N} 3: \mathrm{GU} \& \mathrm{BF} \rightarrow \mathrm{Op} \vee \mathrm{Oq}$ (2); $\mathrm{N} 4: \mathrm{GU} \& \mathrm{BF} \rightarrow \mathrm{Fr}(2) ; \mathrm{N} 5: \mathrm{Gs} \& \mathrm{BF} \rightarrow \mathrm{Fs}(\mathrm{p})(2) ; \mathrm{N} 6: \mathrm{GN} \& \neg \mathrm{Bf} \rightarrow \mathrm{Op}(2) ;$ y N7: $\mathrm{GS} \& \neg \mathrm{BF} \rightarrow \mathrm{Fs}(\mathrm{q})(2)$. La relación $\alpha$ de $\mathrm{S} 1$ expresada como caso-solución tiene 2 propiedades relevantes. Desde un punto de vista lógico y en atención al criterio de mayor generalidad entendida como menor cantidad de propiedades relevantes que las normas del sistema, $\alpha$ es igual de general (o de fino) que las normas N2 a N7 y menos general que N1. Si para considerar que $\alpha$ es un principio implícito de $\mathrm{S} 1$ se atiende a efectos lógicos a su generalidad en contraposición con la generalidad de otras normas del sistema, no resulta justificado entender que $\alpha$ lo es porque su generalidad como enunciado formal es igual a las de las normas N2 a N7 de S1 y menor que la de N1.

Por tanto, desde un análisis mediante lógica deóntica la relación caso-solución $\alpha: \neg \mathrm{GS} \& \mathrm{BF} \rightarrow$ Fr no podría considerarse un principio implícito del sistema $\mathrm{S} 1$ según el criterio de generalidad. Es decir, a efectos lógicos se podría afirmar que $\alpha$ es una consecuencia lógica de las normas que constituyen los axiomas del sistema S1, y por tanto que $\alpha$ es una norma implícita que pertenece a $\mathrm{S} 1$ al igual que pertenecen las normas explícitas. Sin embargo, un análisis lógico no refleja que $\alpha$ tenga la característica de mayor generalidad que las normas $\mathrm{N} 1$ a N7 ${ }^{45}$, por lo que de acuerdo a sus características formales no se podría afirmar que $\alpha$ es un principio implícito de S1.

\section{PRINCIPIOS IMPLÍCITOS Y PESO}

\subsection{Peso y lógica de preferencias}

La característica diferencial de los principios entendida como peso se expresa a través de la lógica de preferencias según examinado en el apartado II. Por tanto, en un

45 De hecho, la norma más general que el resto sería N1, de la cual se podría predicar que constituye el principio del sistema si se atiende a la generalidad. 
análisis formal no será posible verificar el peso del principio $\alpha$ de S1 porque está expresado como correlación caso-solución. En cambio, sí será posible investigar mediante la lógica la dimensión de peso en el resto de principios implícitos examinados por AlONSO en los trabajos citados, esto es, el resto de principios que extrae de los sistemas S1 y S2 y que expresa mediante lógica de preferencias, como ordenación del tratamiento en el sistema a las diferentes propiedades. Tales principios son $\beta$ : $G N \geq G U \geq G S$; $\gamma: \mathrm{BF}>\neg \mathrm{BF} ; \delta$ : BFE $\geq \neg \mathrm{BFE} ; \varepsilon: \mathrm{BFA} \geq \neg \mathrm{BFA}$; $\zeta$ : TO $\geq \neg \mathrm{TO}$.

Sin embargo, del análisis lógico, y partiendo de un punto de vista positivista, surgen problemas para considerar que las relaciones $\beta, \gamma, \delta, \varepsilon$ y $\zeta$ son principios implícitos del sistema por ser relaciones elaboradas de acuerdo con un criterio de preferencia externo. Partiendo del tratamiento del peso como preferencia y dada la definición de propiedad relevante de un sistema normativo, a continuación propongo entender las relaciones de preferencia $\beta, \gamma, \delta, \varepsilon$ y $\zeta$ como una mera consecuencia lógica de la aplicación de un criterio de preferencia, siendo el criterio de preferencia el elemento que incorpora la dimensión de peso. En tal caso, mientras las relaciones entre las normas son necesarias y derivan de la estructura del sistema, el criterio de ordenación es contingente y añade el peso que las normas solas no tenían.

\subsection{Propiedades relevantes y criterio de preferencia}

En el sistema de lógica deóntica de AlCHOURRón y BuLYGIN, una propiedad es relevante en un sistema normativo si, en igualdad del resto de propiedades, los casos en que la propiedad está presente y ausente tienen diferente solución normativa. De esta manera, la propiedad P1 es relevante respecto de los casos P1\&P2\&P3 y $\neg \mathrm{P} 1 \& \mathrm{P} 2 \& \mathrm{P} 3$ si ambos casos tienen diferentes soluciones normativas, por ejemplo P1\&P2\&P3 $\rightarrow \mathrm{Ox}$ $\mathrm{y} \neg \mathrm{P} 1 \& \mathrm{P} 2 \& \mathrm{P} 3 \rightarrow \mathrm{Phx}^{46}$. Dada esta definición de propiedad relevante, si se establece un criterio que ordene la preferencia por las soluciones normativas de un sistema, siempre será posible extraer una relación de orden que manifieste el diferente tratamiento de las propiedades relevantes en dicho sistema, en tanto que la presencia o ausencia de una propiedad relevante siempre tendrán soluciones normativas diferentes y el criterio permitirá ordenarlas. Para demostrarlo, consideraré un sistema hipotético S4 con tres normas N1: $\neg \mathrm{P} 1 \rightarrow \mathrm{Fx}$; N2: $\neg \mathrm{P} 2 \rightarrow \mathrm{Ox}$; y N3: P1\&P2 $\rightarrow \mathrm{Phx}$.

\begin{tabular}{|c|c|c|c|c|c|}
\hline & P1 & P2 & $\mathrm{Fx} / \neg \mathrm{P} 1$ & $\mathrm{Ox} / \neg \mathrm{P} 2$ & $\mathrm{Phx} / \mathrm{P} 1 \& \mathrm{P} 2$ \\
\hline 1 & + & + & & & Phx \\
\hline 2 & - & + & $\mathrm{Fx}$ & & \\
\hline 3 & + & - & & $\mathrm{Ox}$ & \\
\hline 4 & - & - & $F_{x}$ & $\mathrm{Ox}$ & \\
\hline
\end{tabular}

Este sistema S4 tiene en cuenta dos propiedades relevantes (P1 y P2) y una sola acción (x). La presencia o ausencia de P1 y de P2 siempre cambia la solución norma-

46 Alchourrón y Bulygin, 1974: cap. VI. En el ejemplo, x representa una acción cualquiera y P1, P2 y $\mathrm{P} 3$, propiedades relevantes cualquiera. 
tiva. Por tanto, siempre que se tenga un criterio de preferencia será posible ordenar las soluciones normativas a los casos en que concurra una propiedad y en los que no, ceteris paribus. Para realizar el análisis se tomará primero como criterio de preferencia el criterio que prefiere las soluciones que no obliguen a $\mathrm{x}$ frente a las que sí obligan: $\neg \mathrm{Ox}>\mathrm{Ox}{ }^{47}$. Las soluciones normativas en casos en que concurra P1 y $\neg \mathrm{P} 1$ o P2 y $\neg \mathrm{P} 2$ se confrontarán según este criterio, manteniéndose el resto de circunstancias iguales.

\begin{tabular}{|c|c|}
\hline \multicolumn{2}{|c|}{$\mathrm{P} 1$ ? $\neg \mathrm{P} 1^{\mathrm{a}}$ en $\mathrm{S} 4$ según el criterio $\neg \mathrm{Ox}>\mathrm{Ox}$} \\
\hline $\mathrm{P} 1 \& \mathrm{P} 2 ? \neg \mathrm{P} 1 \& \mathrm{P} 2(\neg \mathrm{Ox}>\mathrm{Ox})$ & $\mathrm{P} 1 \& \neg \mathrm{P} 2 ? \neg \mathrm{P} 1 \& \neg \mathrm{P} 2(\neg \mathrm{Ox}>\mathrm{Ox})$ \\
\hline (1)P1\&P2 $\rightarrow \mathrm{Phx} ?(2) \neg \mathrm{P} 1 \& \mathrm{P} 2 \rightarrow \mathrm{Fx}$ & (3)P1\& $\neg \mathrm{P} 2 \rightarrow \mathrm{Ox}$ ?(4) $\neg \mathrm{P} 1 \& \neg \mathrm{P} 2 \rightarrow \mathrm{Fx} \vee \mathrm{Ox}$ \\
\hline $\mathrm{P} 1 \rightarrow \mathrm{Phx} ? \neg \mathrm{P} 1 \rightarrow \mathrm{Fx}$ & $\mathrm{P} 1 \rightarrow \mathrm{Ox} ? \neg \mathrm{P} 1 \rightarrow \mathrm{Fx} \vee \mathrm{Ox}$ \\
\hline$\neg \mathrm{Ox}>\mathrm{Ox}$ & $\neg \mathrm{Ox}>\mathrm{Ox}$ \\
\hline $\mathrm{Phx}=\mathrm{Fx}$ & $\mathrm{Ox} \leq \mathrm{Fx} \vee \mathrm{OX}$ \\
\hline$P 1=\neg P 1$ & $\mathrm{P} 1 \leq \neg \mathrm{P} 1$ \\
\hline \multicolumn{2}{|c|}{$\mathrm{P} 2 ? \neg \mathrm{P2}$ en $\mathrm{S} 4$ según el criterio $\neg \mathrm{Ox}>\mathrm{Ox}$} \\
\hline $\mathrm{P} 1 \& \mathrm{P} 2 ? \mathrm{P} 1 \& \neg \mathrm{P} 2(\neg \mathrm{Ox}>\mathrm{Ox})$ & $\neg \mathrm{P} 1 \& \mathrm{P} 2 ? \neg \mathrm{P} 1 \& \neg \mathrm{P} 2(\neg \mathrm{Ox}>\mathrm{Ox})$ \\
\hline 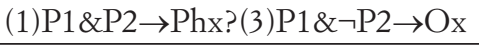 & (2) $\neg \mathrm{P} 1 \& \mathrm{P} 2 \rightarrow \mathrm{Fx} ?(4) \neg \mathrm{P} 1 \& \neg \mathrm{P} 2 \rightarrow \mathrm{Fx} \vee \mathrm{Ox}$ \\
\hline $\mathrm{P} 2 \rightarrow \mathrm{Phx} ? \neg \mathrm{P} 2 \rightarrow \mathrm{Ox}$ & $\mathrm{P} 2 \mathrm{Fx} ? \neg \mathrm{P} 2 \mathrm{Fx} \vee \mathrm{Ox}$ \\
\hline$\neg \mathrm{Ox}>\mathrm{Ox}$ & $\neg \mathrm{Ox}>\mathrm{Ox}$ \\
\hline $\mathrm{Phx}>\mathrm{Ox}$ & $\mathrm{Fx}_{\mathrm{x}} \geq \mathrm{Fx} \vee \mathrm{OX}$ \\
\hline $\mathrm{P} 2>\neg \mathrm{P2}$ & $\mathbf{P} 2 \geq \neg \mathbf{P 2}$ \\
\hline
\end{tabular}

a El símbolo ? representa una relación de preferencia aún por terminar.

De la comparación resulta que en $\mathrm{S} 4$, según el criterio $\neg \mathrm{Ox}>\mathrm{Ox}$, la presencia de $\mathrm{P} 1$ es tratada igual o peor que su presencia $(\mathrm{P} 1 \leq \neg \mathrm{P} 1)$ y la presencia de $\mathrm{P} 2$ es tratada igual o mejor que su ausencia (P2 $\geq \neg \mathrm{P} 2)$.

Por último, es necesario destacar también la importancia esencial que tiene el criterio en este método formal de obtener relaciones y en su resultado. La importancia del criterio se resalta más aún si se invierte el criterio (es decir, sustituyendo $\neg \mathrm{Ox}>\mathrm{Ox}$ por $\neg \mathrm{Ox}<\mathrm{Ox}$ ), porque invirtiendo el criterio las relaciones cambian:

\begin{tabular}{|c|c|}
\hline \multicolumn{2}{|c|}{$\mathrm{P} 1$ ? $\neg \mathrm{P} 1$ en $\mathrm{S} 4$ según el criterio $\neg \mathrm{Ox}<\mathrm{Ox}$} \\
\hline $\mathrm{P} 1 \& \mathrm{P} 2 ? \neg \mathrm{P} 1 \& \mathrm{P} 2(\neg \mathrm{Ox}<\mathrm{Ox})$ & $\mathrm{P} 1 \& \neg \mathrm{P} 2 ? \neg \mathrm{P} 1 \& \neg \mathrm{P} 2(\neg \mathrm{Ox}<\mathrm{Ox})$ \\
\hline (1)P1\&P2 $\rightarrow \mathrm{Phx} ?(2) \neg \mathrm{P} 1 \& \mathrm{P} 2 \rightarrow \mathrm{Fx}$ & (3)P1\& $\neg \mathrm{P} 2 \rightarrow \mathrm{Ox}$ ? (4) $\neg \mathrm{P} 1 \& \neg \mathrm{P} 2 \rightarrow \mathrm{Fx} \vee \mathrm{Ox}$ \\
\hline $\mathrm{P} 1 \rightarrow \mathrm{Phx} ? \neg \mathrm{P} 1 \rightarrow \mathrm{Fx}$ & $\mathrm{P} 1 \rightarrow \mathrm{Ox} ? \neg \mathrm{P} 1 \rightarrow \mathrm{Fx} \vee \mathrm{Ox}$ \\
\hline$\neg \mathrm{Ox}<\mathrm{Ox}$ & $\neg \mathrm{Ox}<\mathrm{Ox}$ \\
\hline $\mathrm{Phx}=\mathrm{Fx}$ & $\mathrm{Ox} \geq \mathrm{Fx} \vee \mathrm{OX}$ \\
\hline$P 1=\neg P 1$ & $\mathbf{P 1} \geq \neg \mathbf{P 1}$ \\
\hline
\end{tabular}

47 Para las cuestiones de justificación de los criterios, vid. apartado 9. 


\begin{tabular}{|c|c|}
\hline \multicolumn{2}{|c|}{$\mathbf{P} 2 ? \neg \mathbf{P} 2$ en $\mathbf{S} 4$ según el criterio $\neg \mathbf{O x}<\mathbf{O x}$} \\
\hline $\mathbf{P} 1 \& \mathbf{P} 2$ ?P1\& $\neg \mathbf{P} 2(\neg \mathbf{O x}<\mathbf{O x})$ & $\neg \mathbf{P} 1 \& \mathbf{P} 2 ? \neg \mathbf{P} 1 \& \neg \mathbf{P} 2(\neg \mathbf{O x}<\mathbf{O x})$ \\
\hline$(1) \mathrm{P} 1 \& \mathrm{P} 2 \rightarrow \mathrm{Phx} ?(3) \mathrm{P} 1 \& \neg \mathrm{P} 2 \rightarrow \mathrm{Ox}$ & $(2) \neg \mathrm{P} 1 \& \mathrm{P} 2 \rightarrow \mathrm{Fx} ?(4) \neg \mathrm{P} 1 \& \neg \mathrm{P} 2 \rightarrow \mathrm{Fx} \vee \mathrm{Ox}$ \\
\hline $\mathrm{P} 2 \rightarrow \mathrm{Phx} ? \neg \mathrm{P} 2 \rightarrow \mathrm{Ox}$ & $\mathrm{P} 2 \mathrm{Fx} ? \neg \mathrm{P} 2 \mathrm{Fx} \vee \mathrm{Ox}$ \\
\hline$\neg \mathrm{Ox}<\mathrm{Ox}$ & $\neg \mathrm{Ox}<\mathrm{Ox}$ \\
\hline $\mathrm{Phx}<\mathrm{Ox}$ & $\mathrm{Fx}<\mathrm{Fx} \vee \mathrm{OX}$ \\
\hline $\mathbf{P} 2<\neg \mathbf{P} 2$ & $\mathbf{P} 2<\neg \mathbf{P 2}$ \\
\hline
\end{tabular}

Una vez invertido el criterio, de la comparación resulta que en S4, según el criterio $\neg \mathrm{Ox}<\mathrm{Ox}$, la presencia de $\mathrm{P} 1$ es tratada igual o mejor que su ausencia $(\mathrm{P} 1 \geq \neg \mathrm{P} 1)$ y la presencia de $\mathrm{P} 2$ es tratada igual o peor que su ausencia $(\mathrm{P} 2 \geq \neg \mathrm{P} 2)$.

\subsection{Las relaciones en $\mathrm{S} 1, \mathrm{~S} 2$ y $\mathrm{S}$ 3, criterio de preferencia y lagunas normativas}

De igual modo que para el hipotético sistema $\mathrm{S} 4$, si se cuenta con un criterio de preferencia será también posible ordenar en S1, S2 y S3 el tratamiento de los casos en que cada una de las propiedades está o no presente ${ }^{48}$.

En S1, según el criterio $O p>\neg(O p)$, la presencia de GN es tratada mejor que su ausencia, la presencia de GU es tratada igual que su ausencia y la presencia de GS es tratada igual que su ausencia. Además, de la comparación de GN, GU y GS en S1 según el criterio $\mathrm{Op}>\neg(\mathrm{Op})$ resulta que cuando concurre BF la presencia de GN es tratada igual o mejor que la de $G U$ y ésta, a su vez, mejor que la de $G S(G N \geq G U>G S)$. Por último, cuando no concurre $\mathrm{BF}$, la presencia de $\mathrm{GN}$ es tratada mejor que la de $\mathrm{GU}$ y ésta, a su vez, igual que la de GS. La conjunción de ambas comparaciones daría lugar a un resultado igual al obtenido por ALONSO, en tanto que la relación se podría enunciar como $\mathrm{GN} \geq \mathrm{GU} \geq \mathrm{GS}$. En S2, según el criterio $\neg(\mathrm{Or})>\mathrm{Or}$, la presencia de BFE es tratada igual o mejor que su ausencia (BFE $\geq \neg B F E$ ). Por último, en $S 3$, según el criterio $\neg(\mathrm{Or})>$ Or, la presencia de BFA es tratada igual o mejor que su ausencia (BFA $\geq \neg \mathrm{BFA})$, la ausencia de $\mathrm{BFE}$ es tratada igual o mejor que su ausencia $(\neg \mathrm{BFE} \geq \mathrm{BFE})$ y la presencia de $\mathrm{TO}$ es tratada igual o mejor que su ausencia $(\mathrm{TO} \geq \neg \mathrm{TO})$.

Podría cuestionarse la aplicación de los criterios de ordenación a los sistemas normativos $\mathrm{S} 1$ y $\mathrm{S} 3$, en tanto que estos sistemas presentan lagunas normativas que no atribuyen una solución normativa a algún caso, mientras que el ejemplo de S4 era un sistema completo. Es decir, podría cuestionarse si el criterio de preferencia puede expresar preferencia también sobre las lagunas normativas. Considero sin embargo que este aspecto no es problemático dada la naturaleza del criterio de ordenación como proposición normativa y las diferencias entre negación interna y externa en el metalenguaje.

En la medida que el criterio de ordenación es extrasistémico, es una proposición normativa. ALCHOURRÓN y BULYGIN ya argumentaron que en las proposiciones nor-

48 Para no interrumpir el argumento del artículo, el desarrollo del sistema de extracción de relaciones en los sistemas S1, S2 y S3 se realiza en el anexo incluido como apartado 11, al cual me remito. 
mativas es posible equiparar la permisión y la ausencia de norma. Esto se debe a que en el lenguaje objeto (normas) solamente es posible la negación interna, mientras que el metalenguaje (proposiciones normativas) admite ambos tipos de negación ${ }^{49}$. Así, en el metalenguaje es posible emplear la negación con un sentido interno y externo. Por este motivo, cuando se enuncia la proposición normativa $\neg \mathrm{Op}$ acerca del sistema S1 hay una ambigüedad. Cuando se usa la negación interna, se afirma que dentro del sistema S1 existe una solución normativa igual a $\neg \mathrm{Op}$, esto es, la proposición afirma que $(\neg \mathrm{Op})$. Sin embargo, cuando se usa la negación externa se afirma que en S1 no existe ninguna solución normativa igual a Op, esto es, la proposición afirma que $\neg(\mathrm{Op})$.

En igual medida, cuando se enuncia la proposición normativa $\neg \mathrm{Op}>\mathrm{Op}$ respecto de S1, se puede entender que en S1 se prefiere la solución normativa $\neg \mathrm{Op}$ sobre la solución normativa $\mathrm{Op}$, esto es, $(\neg \mathrm{Op})>\mathrm{Op}$ —negación interna—, pero también se puede entender que en S1 se prefiere la ausencia de la solución normativa Op a su presencia, esto es $\neg(\mathrm{Op})>\mathrm{Op}$ —negación externa—. Cuando se usa la negación externa para la proposición normativa que actúa como criterio, $\neg(\mathrm{Op})$ es verdadera cuando en S1 las soluciones normativas son Fp o Php, y también cuando no hay solución normativa, es decir, cuando hay una laguna normativa. En igual medida, cuando se emplea la negación externa, la proposición normativa $\neg(\mathrm{Op})>\mathrm{Op}$ respecto de $\mathrm{S} 1$ expresa que en $\mathrm{S} 1$ se prefiere la ausencia de la solución normativa $\mathrm{Op}$ a su presencia, incluso cuando esa ausencia se debe a que el sistema no establece ninguna solución normativa para un caso genérico.

En lo sucesivo, en este trabajo la negación usada en proposiciones normativas siempre se entenderá como negación externa, y para ello se colocará fuera del paréntesis. Al adoptar como criterio de ordenación de preferencias una proposición normativa descriptiva con negación externa, desaparece el problema para ordenar soluciones normativas en sistemas incluso cuando presenten lagunas normativas, lo cual salva la objeción respecto los sistemas $\mathrm{S} 1$ y S3 ${ }^{50}$.

\section{LOS PRINCIPIOS COMO ORDENACIÓN DE PREFERENCIAS}

Como se ha argumentado, siempre que se tenga un criterio de preferencia, mediante un análisis lógico será posible extraer en cualquier sistema normativo las relaciones entre las soluciones de dos casos sólo diferenciados por la concurrencia o no concurrencia de una propiedad relevante. Estas relaciones tendrán carácter necesario y pertenecerán a la propia estructura de un sistema normativo con propiedades relevantes. Ello afecta a la concepción de las relaciones como principios jurídicos implícitos. Aunque la propuesta de ALONSO es muy fructífera a la hora de revelar las relaciones de un sistema normativo, el análisis lógico no permite afirmar que tales relaciones son principios jurídicos.

49 Vid. Alchourrón y Bulygin, 1974: cap. VII. También en Alchourrón y Bulygin, 1991.

50 Las antinomias no presentan tantos problemas. Para tratarlas, las soluciones normativas para cada caso deberían considerarse en su conjunto, de manera que en caso de antinomia se aplicará el criterio de preferencia sobre la conjunción de las soluciones normativas que el sistema establece para cada caso genérico. Así, de acuerdo con el criterio $\neg \mathrm{Ox}>\mathrm{Ox}$ antes escogido, la antinomia $\mathrm{Ox} \& \mathrm{Fx}$ será igual de preferida que Ox pero menos que $\mathrm{Fx}_{\mathrm{x}}(\mathrm{Fx}>\mathrm{Ox}=\mathrm{Fx} \& \mathrm{Ox})$. 
Según se expuso, la lógica de preferencias permite representar el peso de los principios jurídicos. Además, también se planteó que las relaciones expresadas lógicamente mediante un criterio de preferencia son consecuencias necesarias de la existencia de un criterio, pero que el peso expresado como preferencia está contenido en el criterio, no en las relaciones. Es decir, el análisis lógico revela que las relaciones entre las soluciones normativas adquieren el peso al aplicarse el criterio de preferencia, pero no tienen peso propio. Por tanto, una aproximación formal revela que el principio sería el criterio (que contiene la preferencia), no las relaciones sistemáticas (que sólo la aplican). De ello se deriva que a efectos lógicos las relaciones sistemáticas propuestas por ALONSO se podrían considerar sólo consecuencias lógicas de dicho principio, pero no principios.

La aplicación del principio (criterio de ordenación) a un sistema permitiría explicitar la estructura del sistema de acuerdo con dicho principio u orden de preferencias entre soluciones, pero esas preferencias en el tratamiento de propiedades no podrían considerarse principios sino meras consecuencias lógicas de la aplicación del principio. Así pues, se podría decir que la estructura lógica del sistema S1 bajo el principio $\mathrm{Op}>\neg(\mathrm{Op})$ revela las siguientes relaciones sistemáticas ${ }^{51}$ : i) $\mathrm{GN}>\neg \mathrm{GN}$; ii) $\mathrm{GU}=\neg \mathrm{GU}$; iii) $\mathrm{GS}=\neg \mathrm{GS}$; iv) $\mathrm{GN} \geq \mathrm{GU}>\mathrm{GS}$ (ceteris paribus $\mathrm{BF}$ ), y v) $\mathrm{GN}>\mathrm{GU}>\mathrm{GS}$ (ceteris paribus $\neg \mathrm{BF}$ ). Sin embargo, no se puede considerar que dichas consecuencias sean lógicamente principios porque si cambiara el criterio, el orden expresado cambiaría.

Todavía podría hacerse otra objeción para considerar las relaciones como principios implícitos. En un sistema normativo, las consecuencias lógicas de las normas son normas implícitas que pertenecen al sistema normativo. Análogamente, se podría afirmar que las consecuencias lógicas de un principio usado como criterio de preferencia son principios implícitos que pertenecen al sistema normativo ${ }^{52}$. Este paralelismo tropieza con una gran piedra. Las normas que actúan como axiomas en un sistema normativo están formuladas expresamente. Por el contrario, los criterios de ordenación usados no siempre se encuentran en el sistema, sino que pueden ser elementos extrasistémicos añadidos para revelar las relaciones sistemáticas. Para que las consecuencias de un criterio sean principios jurídicos implícitos del sistema, primero habrá que justificar que el criterio es un principio jurídico implícito del sistema. Por tanto, el mero paralelismo con las normas y las consecuencias lógicas de las normas no es suficiente para defender que las relaciones sistemáticas de preferencia son principios implícitos de un sistema normativo.

En todo caso, estoy de acuerdo en que existe una conexión, ya apuntada por otros autores, entre principios implícitos e intereses o valores y criterio de ordenación. ATIENZA y RUIZ MANERO han sostenido, sobre la justificación de los principios implícitos como premisas, que «afirmar que "X es un principio implícito" de una determinada institución, sector normativo o sistema jurídico es lo mismo que afirmar que las reglas y principios explícitos correspondientes son coherentes con $\mathrm{X}$ y que a tales reglas y principios explícitos, entendidos como formulaciones lingüísticas, debe adscribírseles un contenido proposicional que resulte coherente con $\mathrm{X} »^{53}$. Esta reflexión se podría

\footnotetext{
51 Para el significado de esas relaciones sistemáticas vid. apartado 6.3.

52 Agradezco a Juan Pablo AlONSO que me indicara esta objeción.

53 Atienza y Ruiz Manero, 1996: 18-19 y 24.
} 
acomodar a la propuesta de considerar el criterio de elección como principio, lo que exigiría entender las relaciones entre reglas y principios de acuerdo con el principio. En todo caso, esta reflexión también resta importancia a las relaciones sistemáticas.

\section{PRINCIPIOS JURÍDICOS, NORMAS Y PROPOSICIONES NORMATIVAS}

\subsection{Coherencia como adecuación de normas a un principio}

Lo expuesto hasta ahora tiene importantes consecuencias cuando se aplica al concepto de coherencia que usa ALONSO (siguiendo a MACCORMICK) como adecuación de normas a un principio ${ }^{54}$. Si las relaciones entre normas son una consecuencia necesaria de la existencia de un criterio de ordenación, todos los sistemas son necesariamente coherentes con cualquier principio que se proponga como criterio de preferencia, y siempre será posible extraer como mínimo tantas relaciones como combinaciones sean posibles manteniendo igual una propiedad para cumplir con la condición ceteris paribus ${ }^{55}$. Sin embargo, aunque resulta contraintuitivo afirmar que todos los sistemas jurídicos son coherentes según el concepto de coherencia usado por ALONSO, este problema desaparece si se analiza a la luz de las diferencias entre principios entendidos como normas y principios entendidos como proposiciones normativas.

Cuando se emplea como criterio de ordenación una proposición normativa ajena al sistema de normas, el criterio pertenece al metalenguaje. Esto conlleva: i) que la negación en el criterio se puede usar como negación externa, lo que permite ordenar las relaciones del sistema incluso si presenta lagunas; ii) que las relaciones explicitadas según el criterio no serán consecuencias lógicas del sistema sino consecuencias lógicas de una proposición normativa del metalenguaje derivadas de las características estructurales del sistema, por lo que serían proposiciones normativas a su vez y pertenecerían al, llamémoslo, metasistema, y iii) que al pertenecer al metalenguaje, estas proposiciones normativas serán sólo descriptivas, carecerán de fuerza prescriptiva, lo cual impedirá usarlas o usar el criterio para sugerir modificaciones del sistema (como, por ejemplo, colmar lagunas normativas).

No sucede lo mismo cuando se emplea un principio explícito como criterio de ordenación. Por ejemplo, ALEXY alude a los principios P1: «[d]erecho a la vida y a la integridad física» y P2: «aplicación del derecho penal». De estos principios extrae dos «consecuencias como juicios concretos de deber ser jurídico» que son para P1 «[e] stá prohibido realizar la audiencia oral» y para $\mathrm{P} 2$ «está ordenado realizar la audiencia oral» ${ }^{56}$. En este caso, el sistema normativo contiene un principio que, según postula ALEXY, se puede formular como una preferencia entre soluciones normativas. Por tanto, el principio es una norma prescriptiva que pertenece al sistema. Al usar el principio como criterio de ordenación: i) la negación en ese principio será negación interna, lo

54 AlONSO, 2013: 358. Aunque sólo trato el concepto empleado por AlONSO, el debate sobre la coherencia en relación con la teoría de la interpretación jurídica es mucho más amplio y ha generado un gran interés en las últimas décadas. Para una revisión del estado actual de la cuestión, vid. DiCKSON, 2014.

55 El número total de relaciones para un sistema será de $2^{\mathrm{n}}$, es decir, igual al número de casos.

56 Alexy, 1993: 92. 
cual no permitirá incluir las lagunas en la ordenación y permitirá que el sistema sea incoherente con el principio; ii) las relaciones explicitadas serán una consecuencia lógica de una norma del sistema, por tanto serán normas y no proposiciones normativas, y iii) el criterio, como norma, será prescriptivo y permitirá sugerir modificaciones para incrementar la coherencia del sistema.

La diferencia entre principios como normas o como proposiciones normativas revela que la coherencia en el sentido empleado por ALONSO solamente es útil cuando se refiere a principios explícitos que pertenecen al sistema. En este caso, la coherencia tiene carácter contingente y fuerza prescriptiva para corregir defectos del sistema. Sin embargo, cuando la coherencia se refiere a principios ajenos al sistema, los principios son proposiciones normativas y la coherencia es una necesidad lógica. Esta coherencia es trivial, siempre existirá para todos los sistemas normativos pero no servirá para evaluar los sistemas normativos ni para corregir sus defectos.

\subsection{Principios implícitos como proposiciones normativas}

Por otra parte, según la distinción de AlCHOURRÓN y BuLYGIN entre normas (con fuerza prescriptiva jurídica) y proposiciones normativas (sin fuerza prescriptiva), surgen otras consecuencias contraintuitivas de considerar los principios implícitos como proposiciones normativas que se sitúan al nivel del metalenguaje. Estas consecuencias se relacionan con reflexiones ya hechas al examinar en general los principios implícitos en el apartado 3.

La primera consecuencia contraintuitiva es que, dentro del paradigma lógico positivo, los principios implícitos tendrían diferente naturaleza que las normas del sistema, porque los principios son proposiciones normativas, no normas. Por tanto, aunque las normas se consideraran reglas no podrían contraponerse a los principios, lo cual obliga a descartar la tesis de la separación débil respecto de los principios implícitos. Los principios implícitos y las reglas quedarían separados por una diferencia lógica, porque los principios implícitos tendrían una dimensión de peso distinta que sería consecuencia de ser proposiciones normativas pertenecientes al metalenguaje expresadas mediante lógica de preferencias, mientras que las reglas serían normas del lenguaje objeto sin dicha dimensión de peso.

Sin embargo, lo anterior no respalda los argumentos de la tesis de la separación fuerte entre principios y reglas cuando se aplican a los principios implícitos, según se expone a continuación. La segunda conclusión contraintuitiva de considerar que los principios implícitos son proposiciones normativas (de acuerdo con el paradigma lógico positivo de AlCHOURRÓn y BuLYGIN adoptado por AlONSO) es que los principios implícitos no son principios jurídicos, porque no son normas sino proposiciones normativas. Esto hace que los principios implícitos carezcan de fuerza prescriptiva jurídica y que para justificar su normatividad se deba acudir a elementos extrajurídicos. Además, también hace contradictoria la expresión principios implícitos para aludir a los criterios de preferencia no explicitados en el sistema, porque estos criterios, considerados principios implícitos, no pertenecerían al sistema sino que se situarían fuera del mismo como metasistema. 
En relación con los principios implícitos como proposiciones normativas también cabe mencionar la postura de ÁviLA, quien además de considerar las reglas y los principios jurídicos, en su teoría jurídica añade los postulados normativos, que concibe como metanormas cuyo papel es contribuir al entendimiento y aplicación de las normas (por ejemplo, coherencia, razonabilidad y proporcionalidad) ${ }^{57}$. Los postulados según ÁviLA no se consideran reglas ni principios, pues pertenecen al metalenguaje en lugar de al lenguaje objeto. Aunque también se ubiquen en el metalenguaje, los postulados normativos de ÁviLA no son equiparables a los principios según tratados en este trabajo, aunque sería posible indagar qué relaciones existen entre los criterios de ordenación de preferencias y los postulados normativos, cuestión que no abordo en este trabajo.

\section{JUSTIFICACIÓN Y JURIDICIDAD DEL CRITERIO DE PREFERENCIA}

Por último es preciso aludir a la justificación de los criterios de preferencia. La justificación de los criterios de ordenación resulta esencial porque estos criterios no están formulados expresamente, sino que son aspectos extrasistemáticos que se incorporan al razonamiento jurídico. Ello hace aplicable la observación de ALCHOURRÓN respecto de la premisa implícita en el argumento analógico o a pari, la cual «nunca es verdadera por razones lógicas; lo que allí se establece es una pauta valorativa contingente» ${ }^{58}$.

Hay que dejar claro en primer lugar que la ausencia de justificación de la premisa no invalida el razonamiento. La justificación de las premisas de un razonamiento que emplee un criterio de ordenación de preferencias es un problema diferente al de la validez de dicho razonamiento ${ }^{59}$ y no conlleva necesariamente su arbitrariedad. No obstante, como afirma AMAYA, se exige responsabilidad epistémica al operador jurídico en la justificación de su elección, por lo que para poder determinar la responsabilidad del operador éste debería adecuarse a estándares de responsabilidad en la selección de las premisas y explicitar el razonamiento seguido para permitir que se valore su adecuación a dichos estándares ${ }^{60}$.

A efectos de explicitar los razonamientos implícitos en la elección del criterio de ordenación y de acuerdo con lo desarrollado hasta aquí, para que los diferentes criterios de preferencia empleados para ordenar las relaciones sistemáticas en los sistemas S1 a S4 puedan considerarse principios jurídicos sería necesario comprobar si tales principios pertenecen al propio sistema normativo. Recordemos aquí que PRIETO distinguía según el respaldo institucional de los principios jurídicos ${ }^{61}$, por lo que acogiendo su distinción podrían existir principios jurídicos como re-

57 Ávila, 2007: 85 y ss.

58 AlCHOURRÓN, 1991: 13.

59 Moreso, Redondo y Navarro, 1992. Vid. también HaAck, 2007: 19, Bulygin, 2008: 5, y Maranhão, 2009: 232

60 AmAYA, 2011: 10.

61 Vid. apartado 3 anterior. 
laciones de preferencia positivadas en el ordenamiento al modo de los mandatos de optimización de ALEXY (principios explícitos) y por otro lado principios jurídicos no positivados, que serían construcciones valorativas de orden social, moral o práctico, incluso aunque se apoyaran también en consideraciones legales (principios extrasistémicos) ${ }^{62}$.

Como he indicado en el apartado anterior, es posible usar principios explícitos como criterio de preferencia para determinar las relaciones sistemáticas, lo cual no plantea problemas de justificación aunque tiene consecuencias diferentes al uso de principios no explícitos. Sin embargo, la admisibilidad de principios extrasistémicos depende de que la teoría jurídica adoptada establezca o no una separación estricta entre derecho y moral (lo cual remite a los debates HART-DwORKIN y la copiosa literatura surgida sobre la separación de derecho y moral y la adjudicación judicial).

En la teoría positivista de ALCHOURRÓN y BuLYGIN, las normas tienen fuerza prescriptiva (normatividad jurídica) mientras que las proposiciones normativas son descriptivas, al menos a efectos jurídicos ${ }^{63}$. En todo caso, esta distinción no elimina la posible fuerza prescriptiva de las proposiciones normativas, sólo niega su normatividad de carácter jurídico. Junto al derecho coexisten otras fuentes de normatividad además de la jurídica, como son la racionalidad, la moral, la prudencia o el protocolo ${ }^{64}$. Estas fuentes alternativas de normatividad permiten conservar la fuerza prescriptiva no jurídica de las proposiciones normativas en las posturas que separen derecho y moral de manera estricta.

Además, el problema de la ausencia de fuerza prescriptiva de origen jurídico en los principios entendidos como relaciones de preferencia basados en un criterio de ordenación externo no se plantea en otras teorías jurídicas como, por ejemplo, la de DWORKIN ${ }^{65}$. Según la propuesta interpretativa de DWORKIN, la práctica de la interpretación permite revelar el derecho «en su mejor luz» de acuerdo con consideraciones morales o políticos. En la práctica interpretativa, dichos elementos morales o políticos se consideran parte del derecho. Por tanto, los criterios de ordenación procedentes de consideraciones morales y políticas empleadas en la interpretación son en la teoría de DWORKIN parte del sistema normativo, parte del lenguaje objeto (normas jurídicas) y no externos (metalenguaje) ${ }^{66}$. Así pues, con DwORKIN como punto de partida las rela-

62 La construcción de tales principios extrasistémicos se podría justificar como un conjunto de inferencias válidas aceptadas por la comunidad jurídica, aunque explorar las posibilidades explicativas del inferencialismo para explicar el razonamiento con principios excede el propósito de este trabajo.

63 Vid. apartado 3.

64 Vid. BROOME, 2013, 25-27. Agradezco a Miranda DEL CORRAL sus sugerencias de bibliografía sobre normatividad.

65 Doy las gracias al revisor anónimo cuyos comentarios me hicieron ser consciente de este punto.

66 DwORKIN, 1986: 255-256: «Hard cases arise, for any judge, when his threshold test does not discriminate between two or more interpretations of some statute or line of cases. Then be must choose between eligible interpretations by asking which shows the community's structure of institutions and decisions -its public standards as a whole - in a better Light from the stand point of political morality. His own moral and political convictions are now directly engaged. But the political judgment be must make is itself complex and will sometimes set one department of his political morality against another: bis decision will reflect not only bis opinions about justice and fairness but his higher-order convictions about how these ideals should be compromised when they compete». Vid. también DwORKIN, 1978: cap. 4. 
ciones entre normas enunciadas de acuerdo con criterios de ordenación morales o políticos conservan fuerza prescriptiva jurídica porque pertenecen al sistema normativo una vez interpretado. ALONSO podría pues conservar la fuerza prescriptiva jurídica de los principios extrasistémicos abandonando la concepción iuspositivista estricta que sostiene ${ }^{67} \mathrm{y}$ acogiendo una postura más cercana a DwORKIN.

Por último, también plantea problemas la aceptación de principios jurídicos inexpresos pero pertenecientes al sistema, es decir, principios elaborados a partir de material normativo preexistente y que, por tanto, se entienden como principios que pertenecen al sistema (como normas, no como proposiciones normativas). El propio PRIETO parece descartar la posibilidad de que los principios implícitos sean normas, pues reconoce que la elaboración por el intérprete conllevaría «elecciones u opciones valorativas», es decir, «ingredientes extrasistemáticos» ${ }^{68}$. Se podría seguir considerando que los principios implícitos son normas en la concepción de PRIETO si se entiende sistema normativo al modo de AlCHOURRÓn y BULYGIN, es decir, como un sector definido del ordenamiento con un número limitado de normas. En tal caso, los principios inexpresos pero implícitos solamente serían inexpresos en cuanto al sistema, pero serían expresos en algún otro sector del ordenamiento desde el cual se importarían y serían, por tanto, normas. Sin embargo, si se entiende sistema como ordenamiento vigente, según la concepción de PRIETO los principios inexpresos pero implícitos no serían normas, puesto que la mención al uso de elementos extrasistémicos significaría aceptar criterios no jurídicos en el razonamiento, lo cual convertiría los principios en proposiciones normativas.

Es posible una interpretación alternativa para mantener la categoría de principios inexpresos pero implícitos. Esta interpretación se relacionaría con el ejemplo de AleXY ya mencionado, en el que existían dos principios explícitos, P1: «[d]erecho a la vida y a la integridad física» y P2: «aplicación del derecho penal», de los cuales se que extraían dos «consecuencias como juicios concretos de deber ser jurídico», consecuencias que son para P1 «[e]stá prohibido realizar la audiencia oral» y para P2 «está ordenado realizar la audiencia oral» ${ }^{69}$. En estos casos, el razonamiento jurídico aplicaría no un principio explícito, sino las consecuencias lógicas del mismo, es decir, un juicio concreto de deber ser jurídico derivado del principio. Se podría entender que el principio es un principio explícito cuando permita usar su formulación sin más como criterio de preferencia para extraer las relaciones sistemáticas y que, por el contrario, es un principio inexpreso pero implícito cuando sea una consecuencia lógica de un principio expreso determinada por el intérprete.

67 AlONSO, 2014: 383: «El carácter juspositivista del modelo estriba en que el contenido de los principios implícitos viene determinado exclusivamente por el contenido de las normas usadas para obtenerlos. Para la configuración de los principios implícitos no se ha considerado ningún contenido externo al sistema de normas de referencia. Las limitaciones del modelo presentado (en el sentido de que en algunos casos no se puede arribar a una única respuesta correcta) no obstan a que pueda afirmarse que es incorrecto sostener que el juspositivismo es incapaz de dar cuenta del fenómeno de los principios jurídicos implícitos». De hecho, aunque ALONSO reconoce que «de algún modo» los principios como relaciones de orden son valoraciones, considera que no son valoraciones externas a los sistemas normativos en cuestión, sino internas a ellos (384).

68 PRIETO SANCHÍs, 1992: 146.

69 AleXy, 1993: 92. 


\section{CONCLUSIÓN}

Parece que los principios jurídicos implícitos son todavía un misterioso vecino al que la doctrina se encuentra con frecuencia pero del que se sabe poco. El debate sobre principios y reglas ha tratado con mayor asiduidad los principios explícitos que los implícitos, por lo que estos últimos siguen presentando zonas oscuras.

Los resultados de aplicar procedimientos lógicos a los principios implícitos, tal y como ha hecho muy fructíferamente Juan Pablo AlOnso, desvelan ciertas estructuras argumentales pero arrojan dudas razonables sobre lo principios implícitos y abren posibles caminos de investigación. Las tesis sostenidas en torno a la relación entre principios y reglas no son conciliables completamente con la reconstrucción lógica del razonamiento sobre principios implícitos, en especial desde una perspectiva positivista. $\mathrm{Si}$, como parece, los principios implícitos siempre exigen aportar un criterio de preferencia a un sistema normativo, en una teoría que separe derecho y otras fuentes de normatividad, en especial la moral, y que atribuya diferente carácter prescriptivo o descriptivo a lenguaje y metalenguaje, ese criterio y sus consecuencias lógicas se situarían fuera del sistema, lo cual no permitiría contraponer los principios implícitos a las reglas del sistema y arrebataría a los principios implícitos de la nota de juridicidad porque esos criterios de preferencia y sus consecuencias serían metajurídicos, no jurídicos. A efectos lógicos no se habría logrado situar los principios dentro de los sistemas jurídicos como normas, sino que habrían quedado aún a las puertas del derecho.

Podría ser que todas estas confusiones sean consecuencia de mera tozudez semántica. Al hablar de principios implícitos, parecería que se tiene intención de atribuirles las mismas características de los principios explícitos. Si por el contrario se sostuviera que los principios implícitos tienen una categoría normativa diferente a los principios expresos y que la argumentación sobre principios implícitos no es tampoco equivalente a la argumentación sobre principios expresos, se reconocería a los principios implícitos como un tertius genus a investigar sin quedar vinculado por los resultados de investigaciones previas sobre principios y reglas.

\section{ANEXO I: DESARROLLO COMPLETO DE LAS PROPIEDADES DE S1, S2 Y S3 ${ }^{70}$}

Relaciones en S1: según 4.1(ii), el criterio original empleado por ALONSO comprendía: i) $\mathrm{Op} \geq \mathrm{Op} \vee \mathrm{Oq}$; ii) $(\mathrm{Op} \vee \mathrm{Oq}) \& \mathrm{Fr}>\mathrm{Fs}[\mathrm{p}]$, y iii) $\mathrm{Op} \geq \mathrm{Fs}[\mathrm{q}]$. Estos tres criterios se pueden simplificar como $\mathrm{Op}>\neg(\mathrm{Op})$ a efectos de trabajo sin modificar los resultados obtenidos. Con este criterio se ordenan las soluciones normativas a los casos con presencia o ausencia de una propiedad relevante ceteris paribus. Para ello, se añade el caso genérico 7 que garantiza preservar la condición ceteris paribus.

70 Este anexo emplea la notación ya explicada en el trabajo. Como única novedad, LN representa una laguna normativa. 


\begin{tabular}{|c|c|c|c|c|c|c|c|c|c|c|c|}
\hline & GN & $\mathrm{GU}$ & GS & BF & $\mathrm{Op} / \mathrm{GN}$ & $\mathrm{Fr} / \mathrm{GN} \& \mathrm{BF}$ & $\mathrm{Op \vee Oq} / \mathrm{GU} \& \mathrm{BF}$ & $\mathrm{Fr} / \mathrm{GU} \& \mathrm{BF}$ & $\mathrm{Fs}(\mathrm{p}) / \mathrm{Gs} \& \mathrm{BF}$ & $\mathrm{Op} / \mathrm{GN} \& \neg \mathrm{BF}$ & $\mathrm{Fs}(\mathrm{q}) / \mathrm{GS} \& \neg \mathrm{BF}$ \\
\hline 1 & + & - & - & + & Op & $\mathrm{Fr}$ & & & & & \\
\hline 2 & + & - & - & - & Op & & & & & Op & \\
\hline 3 & - & + & - & + & & & $\mathrm{Op \vee Oq}$ & $\mathrm{Fr}$ & & & \\
\hline 4 & - & + & - & - & & & & & & & \\
\hline 5 & - & - & + & + & & & & & $\mathrm{Fs}(\mathrm{p})$ & & \\
\hline 6 & - & - & + & - & & & & & & & $\mathrm{Fs}(\mathrm{q})$ \\
\hline 7 & - & - & - & - & & & & & & & \\
\hline \multicolumn{12}{|c|}{ 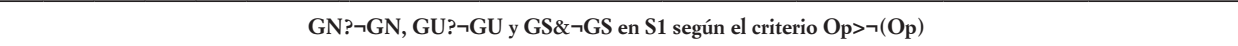 } \\
\hline & & & & & & \multicolumn{6}{|c|}{ Ceteris paribus $\neg \mathrm{GU} \& \neg \mathrm{GS} \& \neg \mathrm{BF}$} \\
\hline & \multicolumn{5}{|c|}{ GN } & \multicolumn{6}{|c|}{ (2) Op } \\
\hline & \multicolumn{5}{|c|}{$\neg \mathrm{GN}$} & \multicolumn{6}{|c|}{ (7) $\mathrm{LN}$} \\
\hline & & & & & & \multicolumn{6}{|c|}{ Ceteris paribus $\neg \mathrm{GN} \& \neg \mathrm{GS} \& \neg \mathrm{BF}$} \\
\hline \multicolumn{6}{|c|}{ GU } & \multicolumn{6}{|c|}{ (4) $\mathrm{LN}$} \\
\hline & \multicolumn{5}{|c|}{$\neg \mathrm{GU}$} & \multicolumn{6}{|c|}{ (7) $\mathrm{LN}$} \\
\hline & & & & & & \multicolumn{6}{|c|}{ Ceteris paribus $\neg \mathrm{GN} \& \neg \mathrm{GU} \& \neg \mathrm{BF}$} \\
\hline \multicolumn{6}{|c|}{ GS } & \multicolumn{6}{|c|}{ (6) Fs $(q)$} \\
\hline \multicolumn{6}{|c|}{$7 \mathrm{GS}$} & \multicolumn{6}{|c|}{ (7) $\mathrm{LN}$} \\
\hline
\end{tabular}

De la comparación resulta que en $\mathrm{S} 1$, según el criterio $\mathrm{Op}>\neg(\mathrm{Op})$, la presencia de GN es tratada mejor que su ausencia, la presencia de GU es tratada igual que su ausencia y la presencia de GS es tratada igual que su ausencia:

$\mathrm{GN} ? \neg \mathrm{GN}$ (ceteris paribus $\neg \mathrm{GU} \& \neg \mathrm{GS} \& \neg \mathrm{BF})$ : Op $>\mathrm{LN}$, por tanto $\mathrm{GN}>\neg \mathrm{GN}$

GU? $\neg \mathrm{GU}$ (ceteris paribus $\neg \mathrm{GN} \& \neg \mathrm{GS} \& \neg \mathrm{BF}): \mathrm{LN}=\mathrm{LN}$, por tanto $\mathrm{GU}=\neg \mathrm{GU}$

GS? $\neg$ GS (ceteris paribus $\neg \mathrm{GN} \& \neg \mathrm{GU} \& \neg \mathrm{BF}): \mathrm{Fs}(\mathrm{q})=\mathrm{LN}$, por tanto $\mathrm{GS}=\neg \mathrm{GS}$

Sin embargo, el principio implícito $\beta$ enunciado por ALONSO era $G N \geq G U \geq G S$, es decir, que el derecho del adquirente era igual o mejor para GN que para GU y este, a su vez, mejor que el de GS. Para comparar simultáneamente las propiedades GN, GU y GS de S1 es necesario mantener la propiedad BF o $\neg \mathrm{BF}$ tal y como hace ALONSO ${ }^{71}$ para preservar el requisito ceteris paribus. De esta manera, para comparar en S4 los resultados relativos a $\mathrm{GN} / \mathrm{GU} / \mathrm{GS}$ respecto de $\mathrm{BF}$ y $\neg \mathrm{BF}$ resulta que:

\begin{tabular}{|c|c|c|}
\hline & Ceteris paribus BF & Ceteris paribus $\neg$ BF \\
\hline GN & $(1) \mathrm{OP} \& F r$ & (2) $\mathrm{Op}$ \\
\hline GU & $(2)(\mathrm{Op} \vee \mathrm{Oq}) \& \mathrm{Fr}$ & (4) $\mathrm{LN}$ \\
\hline GS & $(5) \mathrm{Fs}(\mathrm{p})$ & (6) $\mathrm{Fs}(\mathrm{q})$ \\
\hline
\end{tabular}

GN?GU?GS (ceteris paribus BF): $\mathrm{Op} \& \mathrm{Fr} \geq(\mathrm{Op} \vee \mathrm{Oq}) \& \mathrm{Fr}>\mathrm{Fs}(\mathrm{q})$, por tanto $\mathrm{GN} \geq \mathrm{GU}>\mathrm{GS}$ GN?GU?GS (ceteris paribus $\neg \mathrm{BF}$ ): $\mathrm{Op}>\mathrm{LN}=\mathrm{Fs}(\mathrm{q})$, por tanto $\mathrm{GN}>\mathrm{GU}=\mathrm{GS}$

71 Vid. gráfico 6 en Alonso, 1995: 416. 
De la comparación de GN, GU y GS en S1 según el criterio Op $>\neg(\mathrm{Op})$ resulta que cuando concurre BF la presencia de GN es tratada igual o mejor que la de GU y ésta, a su vez, mejor que la de GS (GN $\geq G U>G S)$. Asimismo, cuando concurre $\neg$ BF la presencia de GN es tratada mejor que la de GU y ésta, a su vez, igual que la de GS. La conjunción de ambas comparaciones daría lugar a un resultado igual al obtenido por ALONSO, en tanto que la relación se podría enunciar como $G N \geq G U \geq G S$.

Relaciones en S2: las relaciones en S2 según el criterio $\neg(\mathrm{Or})>\mathrm{Or}$ son:

\begin{tabular}{|c|c|c|c|c|c|c|c|}
\hline & BFE & BFA & TO & $\mathrm{Or} / \neg \mathrm{BFE}$ & $\mathrm{Or} / \neg \mathrm{BFA}$ & Or/ᄀTO & Fr/BFE\&BFA\&TO \\
\hline 1 & + & + & + & & & & $\mathrm{Fr}$ \\
\hline 2 & - & + & + & Or & & & \\
\hline 3 & + & - & + & & Or & & \\
\hline 4 & - & - & + & Or & Or & & \\
\hline 5 & + & + & - & & & Or & \\
\hline 6 & - & + & - & Or & & Or & \\
\hline 7 & + & - & - & & Or & Or & \\
\hline 8 & - & - & - & Or & Or & Or & \\
\hline \multicolumn{8}{|c|}{ BFE? $\neg$ BFE, BFA? $\neg$ BFA y TO? $\neg$ TO en $S 3$ según el criterio $\neg(\mathrm{Or})>\mathrm{Or}$} \\
\hline & & \multicolumn{2}{|c|}{ C.P. BFA\&TO } & \multicolumn{2}{|c|}{ C.P. BFA\&ᄀTO } & C.P. ᄀBFA\&TO & C.P. $\neg$ BFA\& $\neg$ TO \\
\hline \multicolumn{2}{|c|}{ BFE } & \multicolumn{2}{|c|}{ (1) $\mathrm{Fr}$} & \multicolumn{2}{|c|}{ (5) $\mathrm{Or}$} & (3) Or & (7) $\mathrm{Or}$ \\
\hline \multicolumn{2}{|c|}{$\neg \mathrm{BFE}$} & \multicolumn{2}{|c|}{ (2) Or } & \multicolumn{2}{|c|}{ (6) Or } & (4) $\mathrm{Or}$ & (8) Or \\
\hline \multicolumn{2}{|c|}{ BFA } & \multicolumn{2}{|c|}{ (1) $\mathrm{Fr}$} & \multicolumn{2}{|c|}{ (5) Or } & (2) $\mathrm{Or}$ & (6) Or \\
\hline \multicolumn{2}{|c|}{$\neg \mathrm{BFA}$} & \multirow{2}{*}{\multicolumn{2}{|c|}{ (3) $\mathrm{Or}$}} & \multicolumn{2}{|c|}{ (7) $\mathrm{Or}$} & (4) $\mathrm{Or}$ & (8) Or \\
\hline \multicolumn{2}{|c|}{ TO } & (1) $\mathrm{Fr}$ & & \multicolumn{2}{|c|}{ (3) Or } & (2) $\mathrm{Or}$ & (4) $\mathrm{Or}$ \\
\hline \multicolumn{2}{|c|}{$\neg$ TO } & \multicolumn{2}{|c|}{ (5) Or } & \multicolumn{2}{|c|}{ (7) Or } & (6) $\mathrm{Or}$ & (8) $\mathrm{Or}$ \\
\hline
\end{tabular}

De la comparación resulta que en S2, según el criterio $\neg(\mathrm{Or})>\mathrm{Or}$, la presencia de $\mathrm{BFE}$ es tratada igual o mejor que su ausencia $(\mathrm{BFE} \geq \neg \mathrm{BFE})$, la presencia de BRA es tratada igual o mejor que su ausencia $(B F A \geq \neg B F A)$ y la presencia de TO es tratada igual o mejor que su ausencia $(\mathrm{TO} \geq \neg \mathrm{TO})$ :

$\mathrm{BFE}$ ? $\neg \mathrm{BFE}$ (ceteris paribus $\mathrm{BFA} \& \mathrm{TO}$ ): $\mathrm{Fr}>\mathrm{Or}$, por tanto $\mathrm{BFE}>\neg \mathrm{BFE}$ $\mathrm{BFE}$ ? $\neg \mathrm{BFE}$ (ceteris paribus $\mathrm{BFA} \& \neg \mathrm{TO}$ ): $\mathrm{Or}=\mathrm{Or}$, por tanto $\mathrm{BFE}=\neg \mathrm{BFE}$ $\mathrm{BFE}$ ? $\neg \mathrm{BFE}$ (ceteris paribus $\neg \mathrm{BFA} \& \mathrm{TO}$ ): $\mathrm{Or}=\mathrm{Or}$, por tanto $\mathrm{BFE}=\neg \mathrm{BFE}$ $\mathrm{BFE}$ ? $\neg \mathrm{BFE}$ (ceteris paribus $\neg \mathrm{BFA} \& \neg \mathrm{TO}$ ): $\mathrm{Or}=\mathrm{Or}$, por tanto $\mathrm{BFE}=\neg \mathrm{BFE}$ $\mathrm{BFA}$ ? $\neg \mathrm{BFA}$ (ceteris paribus $\mathrm{BFE} \& \mathrm{TO}$ ): $\mathrm{Fr}>\mathrm{Or}$, por tanto $\mathrm{BFA}>\neg \mathrm{BFA}$ $\mathrm{BFA}$ ? $\neg \mathrm{BFA}$ (ceteris paribus $\mathrm{BFE} \& \neg \mathrm{TO})$ : $\mathrm{Or}=\mathrm{Or}$, por tanto $\mathrm{BFA}=\neg \mathrm{BFA}$ $\mathrm{BFA}$ ? $\neg \mathrm{BFA}$ (ceteris paribus $\neg \mathrm{BFE} \& \mathrm{TO}$ ): $\mathrm{Or}=\mathrm{Or}$, por tanto $\mathrm{BFA}=\neg \mathrm{BFA}$ $\mathrm{BFA} ? \neg \mathrm{BFA}$ (ceteris paribus $\neg \mathrm{BFE} \& \neg \mathrm{TO}$ ): $\mathrm{Or}=\mathrm{Or}$, por tanto $\mathrm{BFA}=\neg \mathrm{BFA}$

$\mathrm{TO} ? \neg \mathrm{TO}$ (ceteris paribus $\mathrm{BFE} \& \mathrm{BFA})$ : $\mathrm{Fr}>\mathrm{Or}$, por tanto $\mathrm{TO}>\neg \mathrm{TO}$

$\mathrm{TO} ? \neg \mathrm{TO}$ (ceteris paribus $\mathrm{BFE} \& \neg \mathrm{BFA})$ : $\mathrm{Or}=\mathrm{Or}$, por tanto $\mathrm{TO}=\neg \mathrm{TO}$

TO? $\neg \mathrm{TO}$ (ceteris paribus $\neg \mathrm{BFE} \& \mathrm{BFA}$ ): $\mathrm{Or}=\mathrm{Or}$, por tanto $\mathrm{TO}=\neg \mathrm{TO}$ $\mathrm{TO} ? \neg \mathrm{TO}$ (ceteris paribus $\neg \mathrm{BFE} \& \neg \mathrm{BFA}$ ): $\mathrm{Or}=\mathrm{Or}$, por tanto $\mathrm{TO}=\neg \mathrm{TO}$ 
Sería posible además extraer otras relaciones de soluciones según las propiedades, puesto que dado el número de propiedades es posible preservar la condición ceteris paribus manteniendo una de ellas y comparando las otras dos. Por ejemplo se podría examinar las relaciones BFE?BFA, $\neg \mathrm{BFE}$ ?BFA, BFE? $\neg \mathrm{BFA}$ y $\neg \mathrm{BFE}$ ? $\neg \mathrm{BFA}$ según criterio $\neg(\mathrm{Or})>$ Or usando TO y $\neg \mathrm{TO}$ para asegurar la condición ceteris paribus.

\begin{tabular}{|c|c|c|}
\hline & Ceteris paribus TO & Ceteris paribus $\neg$ TO \\
\hline BFE & (1)Fr\&(3)Or & (5)Or\&(7)Or \\
\hline$\neg$ BFE & (2)Or\&(4)Or & $(6)$ Or\&(8)Or \\
\hline BFA & (1)Fr\&(2)Or & (5)Or\&(6)Or \\
\hline$\neg$ BFA & (3)Or\&(4)Or & (7)Or\&(8)Or \\
\hline
\end{tabular}

BFE?BFA (ceteris paribus TO): (1)Fr\&(3)Or=(1)Fr\&(2)Or, por tanto BFE=BFA BFE?BFA (ceteris paribus $\neg \mathrm{TO})$ : (5)Or\&(7)Or=(5)Or\&(6)Or, por tanto BFE=BFA $\neg \mathrm{BFE}$ ?BFA (ceteris paribus TO): (2)Or\&(4)Or $\leq(1) \mathrm{Fr} \&(2) \mathrm{Or}$, por tanto $\neg \mathrm{BFE} \leq \mathrm{BFA}$ $\neg$ BFE?BFA (ceteris paribus $\neg$ TO): (6)Or\&(8)Or=(5)Or\&(6)Or, por tanto $\neg$ BFE=BFA $\mathrm{BFE}$ ? $\neg \mathrm{BFA}$ (ceteris paribus TO): (1)Fr\&(3)Or $\geq(3) \mathrm{Or} \&(4) \mathrm{Or}$, por tanto $\mathrm{BFE} \geq \neg \mathrm{BFA}$ BFE? $\neg$ BFA (ceteris paribus $\neg$ TO): (5)Or\&(7)Or=(7)Or\&(8)Or, por tanto BFE $=\neg \mathrm{BFA}$ $\neg \mathrm{BFE} ? \neg \mathrm{BFA}$ (ceteris paribus TO): (2)Or\&(4)Or $=(3) \mathrm{Or} \&(4) \mathrm{Or}$, por tanto $\neg \mathrm{BFE}=\neg \mathrm{BFA}$

$\neg \mathrm{BFE}$ ? $\neg \mathrm{BFA}$ (ceteris paribus $\neg \mathrm{TO})$ : (6)Or\&(8)Or=(7)Or\&(8)Or, $\neg \mathrm{BFE}=\neg \mathrm{BFA}$

Relaciones en S3: aplicando el criterio de preferencia $\neg(\mathrm{Or})>$ Or también sería posible extraer también las relaciones normativas de S3 aunque ALONSO no las extrajo:

\begin{tabular}{|c|c|c|c|c|c|c|}
\hline & BFE & BFA & TO & $\mathrm{BFE} \& \mathrm{BFA} \& \mathrm{TO} \rightarrow \mathrm{Fr}$ & $\mathrm{BFE} \& \neg \mathrm{BFA} \rightarrow \mathrm{Or}$ & $\neg \mathrm{TO} \rightarrow \mathrm{Or}$ \\
\hline 1 & + & + & + & $\mathrm{Fr}$ & & \\
\hline 2 & - & + & + & & & \\
\hline 3 & + & - & + & & Or & \\
\hline 4 & - & - & + & & & \\
\hline 5 & + & + & - & & & Or \\
\hline 6 & - & + & - & & & Or \\
\hline 7 & + & - & - & & Or & Or \\
\hline 8 & - & - & - & & & Or \\
\hline \multicolumn{7}{|c|}{ BFE? $\neg$ BFE, BFA? $\neg$ BFA y TO? $\neg$ TO en $\mathrm{S} 3$ según el criterio $\neg($ Or $)>($ Or $)$} \\
\hline & & \multicolumn{2}{|c|}{ BFA\&TO } & BFA\&ᄀTO & $\neg \mathrm{BFA \& TO}$ & $\neg \mathrm{BFA \& \neg TO}$ \\
\hline \multicolumn{2}{|c|}{ BFE } & \multicolumn{2}{|c|}{ (1) $\mathrm{Fr}$} & (5) Or & (3) Or & (7) Or \\
\hline \multicolumn{2}{|c|}{$\neg \mathrm{BFE}$} & \multicolumn{2}{|c|}{ (2) LN } & (6) Or & (4) LN & (8) Or \\
\hline \multicolumn{2}{|c|}{ BFA } & \multicolumn{2}{|c|}{ (1) $\mathrm{Fr}$} & (5) Or & (2) LN & (6) $\mathrm{Or}$ \\
\hline \multicolumn{2}{|c|}{$\neg \mathrm{BFA}$} & \multicolumn{2}{|c|}{ (3) Or } & (7) Or & (4) $\mathrm{LN}$ & (8) Or \\
\hline \multicolumn{2}{|c|}{ TO } & \multicolumn{2}{|c|}{ (1) $\mathrm{Fr}$} & (3) $\mathrm{Or}$ & (2) $\mathrm{LN}$ & (4) $\mathrm{LN}$ \\
\hline \multicolumn{2}{|c|}{ ᄀTO } & \multicolumn{2}{|c|}{ (5) Or } & (7) $\mathrm{Or}$ & (6) Or & (8) Or \\
\hline
\end{tabular}


De la comparación resulta que en S3, según el criterio $\neg(\mathrm{Or})>\mathrm{Or}$, la ausencia de $\mathrm{BFE}$ es tratada igual o mejor que su ausencia $(\neg \mathrm{BFE} \geq \mathrm{BFE})$, la presencia de BRA es tratada igual o mejor que su ausencia $(B F A \geq \neg B F A)$ y la presencia de TO es tratada igual o mejor que su ausencia $(\mathrm{TO} \geq \neg \mathrm{TO})$ :

$\mathrm{BFE} ? \neg \mathrm{BFE}$ (ceteris paribus $\mathrm{BFA} \& \mathrm{TO}$ ): $\mathrm{FR}=\mathrm{LN}$, por tanto $\mathrm{BFE}=\neg \mathrm{BFE}$ $\mathrm{BFE}$ ? $\neg \mathrm{BFE}$ (ceteris paribus $\mathrm{BFA} \& \neg \mathrm{TO}$ ): $\mathrm{FR}=\mathrm{LN}$, por tanto $\mathrm{BFE}=\neg \mathrm{BFE}$ $\mathrm{BFE}$ ? $\neg \mathrm{BFE}$ (ceteris paribus $\neg \mathrm{BFA} \& \mathrm{TO}$ ): $\mathrm{Or}<\mathrm{LN}$, por tanto $\mathrm{BFE}<\neg \mathrm{BFE}$ $\mathrm{BFE}$ ? $\neg \mathrm{BFE}$ (ceteris paribus $\neg \mathrm{BFA} \& \neg \mathrm{TO}$ ): Or=Or, por tanto $\mathrm{BFE}=\neg \mathrm{BFE}$ $\mathrm{BFA}$ ? $\neg \mathrm{BFA}$ (ceteris paribus $\mathrm{BFE} \& \mathrm{TO})$ : $\mathrm{Fr}>\mathrm{Or}$, por tanto $\mathrm{BFA}>\neg \mathrm{BFA}$ $\mathrm{BFA}$ ? $\neg \mathrm{BFA}$ (ceteris paribus $\mathrm{BFE} \& \neg \mathrm{TO}$ ): $\mathrm{Or}=\mathrm{Or}$, por tanto $\mathrm{BFA}=\neg \mathrm{BFA}$ $\mathrm{BFA}$ ? $\neg \mathrm{BFA}$ (ceteris paribus $\neg \mathrm{BFE} \& \mathrm{TO}$ ): $\mathrm{LN}=\mathrm{LN}$, por tanto $\mathrm{BFA}=\neg \mathrm{BFA}$ $\mathrm{BFA}$ ? $\neg \mathrm{BFA}$ (ceteris paribus $\neg \mathrm{BFE} \& \neg \mathrm{TO}$ ): $\mathrm{Or}=\mathrm{Or}$, por tanto $\mathrm{BFA}=\neg \mathrm{BFA}$

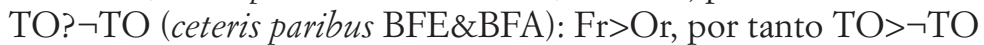
$\mathrm{TO} ? \neg \mathrm{TO}$ (ceteris paribus $\mathrm{BFE} \& \neg \mathrm{BFA})$ : $\mathrm{Or}=\mathrm{Or}$, por tanto $\mathrm{TO}=\neg \mathrm{TO}$ $\mathrm{TO} ? \neg \mathrm{TO}$ (ceteris paribus $\neg \mathrm{BFE} \& \mathrm{BFA}$ ): $\mathrm{LN}>\mathrm{O}$, por tanto $\mathrm{TO}>\neg \mathrm{TO}$ $\mathrm{TO} ? \neg \mathrm{TO}$ (ceteris paribus $\neg \mathrm{BFE} \& \neg \mathrm{BFA})$ : $\mathrm{LN}=\mathrm{Or}$, por tanto $\mathrm{TO}>\neg \mathrm{TO}$

\section{BIBLIOGRAFÍA}

AARNIO, A., 1997: «Las reglas en serio», en La normatividad del derecho, Barcelona: Gedisa, 17-36.

- 2011: Essays on the doctrinal study of law, Dordrecht-Heidelberg-London-New York: Springer, Law and Philosophy Library, núm. 96.

AlCHOURRón, C. E., 1991: «Los argumentos jurídicos a fortiori y a pari», en C. E. AlCHOuRRÓN y E. Bulygin, Análisis Lógico y Derecho, Madrid: Centro de Estudios Constitucionales, 3-24.

AlChourrón, C. E., y Bulygin, E., 1974: Introducción a la metodología de las ciencias jurídicas y sociales (online), Buenos Aires: Astrea. Consultado el 18 de diciembre de 2014. Disponible en bttp://www.biblioteca.org.ar/libros/154933.pdf.

- 1991: «Permisos y normas permisivas», en Análisis Lógico y Derecho, Madrid: Centro de Estudios Constitucionales, 215-238.

ALEXY, R., 1989: A theory of legal argumentation: The theory of rational discourse as theory of legal justification, New York: Oxford University Press.

- 1993: Teoría de los Derechos Fundamentales, E. GARZÓn Valdés (trad.), Madrid: Centro de Estudios Constitucionales.

— 2000: «On the Structure of Legal Principles», Ratio Juris, 13(3), 294-304.

Alonso, J. P., 1995: «Un caso difícil en el Código Civil español», Doxa. Cuadernos de Filosofía del Derecho, 17-18, 403-431.

- 2006: Interpretación de las normas y derecho penal, Buenos Aires: Editores del Puerto.

- 2012, «Modelos jurídicos de Coherencia», Revista Filosofía del Derecho, 1(1), 3-36.

- 2013: «Principios jurídicos implícitos y coherencia», Doxa: Cuadernos de Filosofía del Derecho, 36, 357-386.

AmayA, A., 2011: «Diez tesis acerca de la coherencia en el Derecho», Discusiones, 10, 21-64.

Atienza, M., y Ruiz Manero, J., 1996: Las Piezas del Derecho. Teoría de los Enunciados Jurídicos, Barcelona: Ariel.

ÁvilA, H. B., 2007: Theory of legal principles, Dordrecht-Heidelberg-London-New York: Springer, Law and Philosophy Library, núm. 81. 
Broome, J., 2013: Rationality Through Reasoning, Oxford, Malden MA: Wiley-Blackwell, Blackwell/Brown Lectures in Philosophy.

BuLYGIN, E., 2008: «What can one expect from logic in the law? (Not everything, but more than something: A reply to Susan Haack)», Ratio Juris, 21(1), 150-156.

Comanducci, P., 1998: «Principios jurídicos e indeterminación del derecho», Doxa: Cuadernos de Filosofía del Derecho, 21(2), 89-104.

DicksON, J., 2014: «Interpretation and Coherence in Legal Reasoning», en E. N. ZaLTA (ed.), The Stanford Encyclopedia of Philosophy (Summer 2014 Edition) (online), Stanford: Stanford University (consultado el 3 de marzo de 2015). Disponible en http://plato.stanford.edu/ archives/sum2014/entries/legal-reas-interpret/.

DwORKIN, R. M., 1978: Taking rights seriously (with a new appendix, a response to critics), Cambridge, Mass.: Harvard University Press.

- 1986: Law's empire, Cambridge, Mass.: Harvard University Press.

HAACK, S., 2007: «On logic in the law: Something, but not all», Ratio Juris, 20(1), 1-31.

MARANHÃO, J. S. A., 2009: «La lógica en el Derecho: grandes expectativas y algunas desilusiones», Doxa: Cuadernos de Filosofía del Derecho, 32, 229-254.

Moreso, J. J.; REDONDO, C., y NAVARRO, P. E., 1992: «Argumentación jurídica, lógica y decisión judicial», Doxa: Cuadernos de Filosofía del Derecho, 11, 247-262.

Navarro, P. E., y Rodríguez, J. L., 2014: Deontic Logic and Legal Systems, New York: Cambridge University Press. Cambridge Introductions to Philosophy and Law.

PeczeniK, A., 1992: «Los principios jurídicos según Manuel Atienza y Juan Ruiz Manero», Doxa: Cuadernos de Filosofía del Derecho, 12, 327-331.

PRIETO SANCHís, L., 1992: Sobre principios y normas: problemas del razonamiento jurídico, Madrid: Centro de Estudios Constitucionales.

RAZ, J., 1972: «Legal principles and the limits of law», The Yale Law Journal, 81(5), 823-854.

Ross, A., 1968: Directives and Norms, London: Routledge \& Kegan Paul.

Von Wright, G. H., 1951: «Deontic Logic», Mind, 237(60), 1-15.

- 1963: Norm and Action: A Logical Enquiry, London: Routledge \& Kegan Paul. 